\title{
ISOTOPING MAPPINGS TO OPEN MAPPINGS
}

\author{
BY
}

JOHN J. WALSH

\begin{abstract}
Let $f$ be a quasi-monotone mapping from a compact, connected manifold $M^{m}(m>3)$ onto a space $Y$; then there is an open mapping $g$ from $M$ onto $Y$ such that, for each $y \in Y, g^{-1}(y)$ is not a point and $g^{-1}(y)$ and $f^{-1}(y)$ are equivalently embedded in $M$ (in particular, $g^{-1}(y)$ and $f^{-1}(y)$ have the same shape). Applying the result with $f$ equal to the identity mapping on $M$ yields a continuous decomposition of $M$ into cellular sets each of which is not a point.
\end{abstract}

Let $M^{m}$ be a compact, connected topological manifold of dimension $m>3$; let $f$ be a mapping from $M$ onto a metric space $Y$ satisfying: for each open, connected set $U \subseteq Y$, each component of $f^{-1}(U)$ is mapped onto $U$ by $f$ (such mappings are exactly the quasi-monotone or, equivalently, the quasiopen mappings of Whyburn [Why-1], [Why-2]; in particular, monotone mappings are quasi-open). The main result of this paper is that $f$ can be approximated by an open mapping $g$ from $M$ onto $Y$ satisfying for each $y \in Y$ : (i) $g^{-1}(y)$ is not a point and (ii) $g^{-1}(y)$ and $f^{-1}(y)$ are equivalently embedded in $M$ (in particular, $g^{-1}(y)$ and $f^{-1}(y)$ have the same shape). Even for the case $f=\mathrm{id}_{M}$ (i.e., the identity mapping of $M$ ), this result yields the nontrivial fact that there is a continuous decomposition of $M$ into cellular sets each of which is not a point. Continuous decompositions of the plane into nondegenerate cellular sets were constructed by Anderson [A-4] and Sosinskii [So]. In [A-1], R. D. Anderson announced that the plane can be filled up with a continuous collection of pseudoarcs; however, he never published a proof. The results of this paper were inspired by Theorem II announced by Anderson in [A-1] (Theorem II appears in this paper as Corollary (1.1)).

The techniques used in this paper are reminiscent of those used by Anderson [A-2], [A-3], [A-4], by D. Wilson [Wi-1], [Wi-2], and by Walsh [Wa-1], [Wa-2].

The main result of this paper is stated in $\S 1$; in addition, in $\$ 1$ we show that the main result is a consequence of an apparently weaker result. The proof of this latter result forms the bulk of this paper and is the content of $\$ \$ 5$ and 6; $\$ 5$ contains a technical device and $\$ 6$ contains the actual proof. In $\$ 2$, some

Received by the editors December 3, 1975 and, in revised form, March 11, 1977. AMS (MOS) subject classifications (1970). Primary 54C10; Secondary 57C99.

Key words and phrases. Open mapping, monotone mapping, manifold, cellular. 
fundamental properties of brick partitions are stated; also a filtration obtained from a brick partition is described.

$\S \S 3$ and 4 contain the proof of a very modest version of the main result. These two sections are included strictly for pedagogic reasons; specifically, it is useful to have Proposition (4.2) for comparison when discussing the difficulties faced in proving Proposition (6.1).

Terminology. All spaces considered are assumed to be metric and all spaces of functions are to be given the uniform topology; in particular, for mappings (= continuous functions) between compact spaces the topology is given by the supremum metric. All metrics will be denoted $d(,) . M^{m}$ will denote a compact, connected topological manifold of dimension $m$ possibly with boundary (denoted $\partial M$ ). A set is nondegenerate if it is not a point. Int( ), cl( ), and diam( ) will refer to the topological interior, the closure, and the diameter of a set, respectively. (At times, we will also denote the closure of a set $K$ by $\bar{K}$.) If $A$ is a collection of sets, then $A^{*}$ is the union of members of $A$ and $\mu(A)$, the mesh of $A$, is the supremum of the diameters of the members of $A$. We will abusively use $\cap\left\{A_{\gamma} \mid \gamma \in \Gamma\right\}$ to denote $\bigcap_{\gamma \in \Gamma} A_{\gamma}$.

An ordered collection $\left(A_{1}, \ldots, A_{n}\right)$ is a chain provided $A_{i}=A_{j}$ only if $i=j$ and $A_{i} \cap A_{i-1} \neq \varnothing$ for $i=2, \ldots, n$ (this is not the standard use of the term chain since we are permitting $A_{i} \cap A_{j} \neq \varnothing$ even if $\left.|i-j|>1\right)$. A collection $A$ refines a collection $B$ if each set in $A$ is contained in a set of $B ; A$ is called a refinement of $B$. A sequence $\left\{A_{n}\right\}_{n=1}^{\infty}$ is nested provided $A_{1} \supseteq A_{2} \supseteq$ $A_{3} \ldots$. .

Let $f$ be a mapping from $X$ onto $Y$; $f$ is open provided $f(U)$ is open for each set $U \subseteq X, f$ is monotone provided each $f^{-1}(y)$ is connected, and $f$ is quasi-open or quasi-monotone provided for each open, connected set $V \subseteq Y$ if $U$ is a connected component of $f^{-1}(V)$, then $f(U)=V$ (for mappings between compact, locally connected spaces the above definition of quasi-open and quasi-monotone coincides with standard definition of each; see [Why-2, p. 110] and [Why-1, p. 152]). Two further facts which we need from [Why-2] and [Why-1] are: (i) if $f$ is a quasi-open mapping between compact, locally connected spaces and $f=l \circ m$ is the monotone-light factorization of $f$, then $l$ is open; (ii) if $\left\{f_{n}\right\}_{n=1}^{\infty}$ is a sequence of quasi-open mappings between compact, locally connected spaces and $f=\lim _{n \rightarrow \infty} f_{n}$, then $f$ is quasi-open.

By an isotopy of $X$ we mean a path $\left\{h_{t}\right\}_{t \in[a, b]}$ or $\left\{h_{t}\right\}_{t \in[a, b)}$ of selfhomeomorphisms of $X$ where $[a, b]$ and $[a, b)$ are subintervals of $[0, \infty)$. The statement let $\beta=\beta_{1} \cup \beta_{2} \cup \cdots \cup \beta_{n}$ be an arc will mean that $\beta$ is an arc (we use $\beta$ to denote both the mapping and its image) and the $\beta_{i}$ 's are subarcs with $\beta_{i} \cap \beta_{i+1}$ a single point for $i=1, \ldots, n-1$ and with the $\beta_{i}$ 's covering $\beta ; \beta(0)$ and $\beta_{1}(0)$ (resp., $\beta(1)$ and $\beta_{n}(1)$ ) denote the endpoint of $\beta$ contained in $\beta_{1}$ (resp., $\beta_{n}$ ). 
Let $f$ be a mapping from $X$ onto $Y$; a closed subset $K \subseteq Y$ with $\operatorname{int}(K)=\varnothing$ is called $f$-admissible provided $\operatorname{int}\left(f^{-1}(K)\right)=\varnothing$. We say that $f$ is admissible if each closed subset $K \subseteq Y$ with $\operatorname{int}(K)=\varnothing$ is $f$-admissible (observe that open mappings are admissible).

The support of an isotopy $\left\{h_{t}\right\}_{t \in[a, b]}$ is denoted $\operatorname{supp}\left(\left\{h_{t}\right\}\right)$ and is equal to $\operatorname{cl}\left(\left\{x \mid\right.\right.$ for some $\left.\left.t \in[a, b], h_{t}(x) \neq x\right\}\right)$.

Let $A$ and $B$ be closed subsets of a manifold $M^{m}$; then $A$ and $B$ are equivalently embedded provided there is an isotopy $\left\{h_{t}\right\}_{t \in[0, \infty)}$ of $M$ satisfying: (i) for each neighborhood $V$ of $A$ there is a neighborhood $U$ of $B$ and a $t_{0}$ with $A \subseteq h_{t}(U) \subseteq V$ for all $t \geqslant t_{0}$; (ii) for each neighborhood $U$ of $B$ there is a neighborhood $V$ of $A$ and a $t_{0}$ with $B \subseteq h_{t}^{-1}(V) \subseteq U$ for all $t>t_{0}$.

Let $A$ be a collection of sets and let $B$ be a set; define $\operatorname{st}^{0}(B, A)=\{a \in$ $A \mid a \subseteq B\}, \operatorname{st}^{1}(B, A)=\{a \in A \mid a \cap B \neq \varnothing\}$, and, inductively for $i>2$, $\mathrm{st}^{i}(B, A)=\mathrm{st}^{1}\left(\mathrm{st}^{i-1}(B, A)^{*}, A\right)$; at times we will use $\operatorname{st}(B, A)$ in place of st $^{1}(B, A)$.

1.

MAIN THEOREM. Let $M^{m}$ be a compact, connected manifold with $m>3$, let $f$ be a quasi-open (equiv., quasi-monotone) mapping of $M$ onto a space $Y$, and let $\varepsilon>0$. Then there is an isotopy $\left\{h_{t}\right\}_{t \in[0, \infty)}$ of $M$ satisfying: (i) $h_{0}=$ identity; (ii) $\lim _{t \rightarrow \infty} f \circ h_{t}=g$ exists and $g$ is open; (iii) for each $t \in[0, \infty), d\left(f, f \circ h_{t}\right)<$ $\varepsilon$; (iv) for each $y \in Y$, each component of $g^{-1}(y)$ is nondegenerate and $g^{-1}(y)$ and $f^{-1}(y)$ are equivalently embedded in $M$.

REMARK. It will be clear from the proof of the Main Theorem that if $A$ is a closed subset of $M$ with $\operatorname{dim}(A) \leqslant m-2$ (we are referring to the covering dimension of $A$ ), then we can assume that, for $t \in[0, \infty),\left.h_{t}\right|_{\partial M \cup A}$ equals the identity and, hence, that $\left.f\right|_{\partial M \cup A}=\left.g\right|_{\partial M \cup A}$.

The following corollary was announced by R. D. Anderson [A-1] but a proof was never published.

(1.1) COROLLARY. If $f$ is a monotone mapping from $M^{m}(m>3)$ onto $Y$, then there is a montone open mapping $g$ from $M$ onto $Y$.

REMARK. The author proved a version of (1.1) in [Wa-1, Corollary (3.7.2)] with the additional assumption that $Y$ be a polyhedron; however, the monotone open mapping obtained in [Wa-1] is not obtained by an isotopy of $M$ and definitely does not satisfy condition (iv) in the Main Theorem.

We can quickly reduce the Main Theorem to the case where $f$ is monotone as follows. Let $f=l \circ m$ be the monotone-light factorization of $f$; the Main Theorem applied to the monotone mapping $m$ yields an isotopy of $M$ which also "works" for $f$. 
The remainder of this section deals with showing that the fact that $\lim _{t \rightarrow \infty} f \circ h_{t}=g$ implies that $f^{-1}(y)$ and $g^{-1}(y)$ are equivalently embedded. The proof of the following lemma is left to the reader.

(1.2) Lemma. Let $X$ and $Y$ be compact metric spaces, let $y \in Y$, and let $V$ be a neighborhood of $y$. Then there is an $\varepsilon>0$ such that, for any pair of mappings $f, g$ from $X$ onto $Y$ with $d(f, g)<\varepsilon, g^{-1}(y) \subseteq f^{-1}(V)$.

The following proposition is probably known and is certainly in the spirit contained in [K-L].

(1.3) Proposition. Let $\left\{h_{t}\right\}_{t \in[0, \infty)}$ and $\lim _{t \rightarrow \infty} f \circ h_{t}=g$ be as in the Main Theorem. Let $y \in Y$, let $\left\{W_{i}\right\}_{i=1}^{\infty}$ be a sequence of open neighborhoods of $f^{-1}(y)$ with $\bar{W}_{i} \subseteq W_{i-1}$ and with $f^{-1}(y)=\cap_{i=1}^{\infty} W_{i}$, and let $\varepsilon>0$. Then there is an integer $k$ and a number $s \geqslant 0$ (both depending on $\varepsilon$ and $y$ ) such that $W_{k} \subseteq N_{e}\left(f^{-1}(y)\right)$ and, for each $t>s$,

$$
g^{-1}(y) \subseteq h_{t}^{-1}\left(W_{k}\right) \subseteq N_{e}\left(g^{-1}(y)\right) .
$$

Proof. Let $U \subseteq g\left(N_{\varepsilon}\left(g^{-1}(y)\right)\right)$ be an open neighborhood of $y$ with $g^{-1}(U)$ $\subseteq N_{e}\left(g^{-1}(y)\right)$. Applying (1.2) with $V=U$, there is $t^{\prime}>0$ such that, for $t>t^{\prime},\left(f \circ h_{t}\right)^{-1}(y) \subseteq g^{-1}(U)$. Choose $k$ such that $W_{k} \subseteq N_{e}\left(f^{-1}(y)\right), f\left(W_{k}\right)$ $\subseteq U$, and $\left(f \circ h_{t}\right)^{-1}\left(f\left(W_{k}\right)\right) \subseteq g^{-1}(U)$ for $t>t^{\prime}$. Let $U^{\prime} \subseteq f\left(W_{k}\right)$ be an open neighborhood of $y$ with $f^{-1}\left(U^{\prime}\right) \subseteq W_{k}$. Applying (1.2) with $V=U^{\prime}$, there is $s>t^{\prime}$ such that, for $t>s, g^{-1}(y) \subseteq\left(f \circ h_{t}\right)^{-1}\left(U^{\prime}\right)$. Observe that if $t>s$, then

$$
\begin{aligned}
g^{-1}(y) & \subseteq\left(f \circ h_{t}\right)^{-1}\left(U^{\prime}\right)=h_{t}^{-1}\left(f^{-1}\left(U^{\prime}\right)\right) \subseteq h_{t}^{-1}\left(W_{k}\right) \\
& \subseteq h_{t}^{-1}\left(f^{-1} \circ f\left(W_{k}\right)\right) \subseteq g^{-1}(U) \subseteq N_{\varepsilon}\left(g^{-1}(y)\right) ;
\end{aligned}
$$

hence, the proof is complete.

RemarK. Since $\lim _{t \rightarrow \infty} f \circ h_{t}=g$, it follows that $\lim _{t \rightarrow \infty} g \circ h_{t}^{-1}=f$; applying Proposition (1.3) to the latter yields the conclusion in (1.3) with the roles of $f$ and $g$ interchanged and with $h_{t}^{-1}$ in place of $h_{t}$.

Armed with Proposition (1.3) and this remark it follows easily that $f^{-1}(y)$ and $g^{-1}(y)$ are equivalently embedded in $M$. The purpose of introducing the concept of "equivalently embedded" is to give emphasis to the strong relationship between the point inverses of $g$ and those of $f$; this paper contains no further development of the concept.

2. Throughout this section $Y$ will denote a compact, connected, locally connected metric space. A brick partition $\mathcal{G}$ for $Y$ is a finite collection of pairwise disjoint open connected (nonempty) subsets of $Y$ satisfying: (i) $\mathcal{G}^{*}$ is dense in $Y$ and $g=\operatorname{int}(g)$ for each $g \in \mathcal{G}$; (ii) if $U$ is an open subset of $Y$ and $g, g^{\prime} \in \mathcal{G}$ with $\bar{g} \cap \bar{g}^{\prime} \cap U \neq \varnothing$, then there is a point $y \in \bar{g} \cap \bar{g}^{\prime} \cap U$ 
with $y \in \operatorname{int}\left(\bar{g} \cup \bar{g}^{\prime}\right)$. (We have omitted mention of a metric related condition which the elements of $\mathcal{G}$ must also satisfy; we will not need this condition; see [Bi, p. 304].) By a closed brick partition, we will means a collection consisting of the closures of the elements of a brick partition.

In [Bi], Bing proves that $Y$ has a sequence $\left\{\mathcal{G}_{i}\right\}_{i=1}^{\infty}$ of brick partitions with $\mathcal{G}_{i}$ refining $\mathcal{G}_{i-1}$ and with $\lim _{i \rightarrow \infty} \mu\left(\mathcal{G}_{i}\right)=0$. We will need the following slightly stronger form of this result. Let $f$ be a mapping from a compact metric space $X$ onto $Y$ (we must assume that $Y$ is not a point); a brick partition $\mathcal{G}$ is $f$-admissible provided $\{\bar{g}-g \mid g \in \mathcal{G}\}^{*}$ is $f$-admissible. We assert that for each such mapping $f$ there is a sequence $\left\{\mathcal{G}_{i}\right\}_{i=1}^{\infty}$ of brick partitions for $Y$ as above satisfying the additional requirement that each $\mathcal{G}_{i}$ is $f$-admissible. The proof of this assertion can be extracted from [Bi] by recalling that the collection $W-\left(W_{0}^{\prime} \cup \cdots \cup W_{n}^{\prime}\right)$ constructed in the proof of Theorem 5 on p. 308 of [Bi], is uncountable; this fact makes it possible to choose the brick partitions in Theorems 5-8 in [Bi] to be $f$-admissible.

Given a closed brick partition $\mathcal{G}$ of $Y$, we can obtain a filtration of $Y$ as follows. Let $\mathcal{G}=\left\{g_{1}, \ldots, g_{q}\right\}$; let

$$
\begin{aligned}
\mathbb{Q}=\left\{\left(u_{1}, \ldots, u_{k}\right) \mid 1 \leqslant\right. & u_{1}<u_{2}<\cdots<u_{k} \leqslant q ; g_{u_{1}} \cap \cdots \cap g_{u_{k}} \neq \varnothing ; \\
& \text { if } g_{s} \notin\left\{g_{u_{1}}, \ldots, g_{u_{k}}\right\}, \\
& \text { then } \left.g_{s} \cap\left(g_{u_{1}} \cap \cdots \cap g_{u_{k}}\right) \neq g_{u_{1}} \cap \cdots \cap g_{u_{k}}\right\} .
\end{aligned}
$$

If $x=\left(u_{1}, \ldots, u_{k}\right) \in \mathbb{Q}$ then let $|x|=g_{u_{1}} \cap \cdots \cap g_{u_{k}}$ and call $k$ the length of $x$. Partially order $Q$ by defining $x=\left(u_{1}, \ldots, u_{k}\right) \leqslant x^{\prime}=\left(u_{1}^{\prime}, \ldots, u_{k}^{\prime}\right)$ provided $|x| \subseteq\left|x^{\prime}\right|$; we leave to the reader to check that we have a partial ordering. Let $I(\mathbb{Q})$ be the length of the longest chain in $\mathbb{Q}$; let $\mathfrak{T}_{1}=$ \{minimal elements of $Q$ \}; and, inductively for $t=2, \ldots, I(\mathbb{Q})-1$, let $\mathfrak{R}_{t}=$ \{minimal elements of $\mathbb{Q}-\mathfrak{N}_{t-1}$ which do not have length one $\}$. Let $\Re_{I(\mathbb{Q})}=$ elements in $\mathbb{Q}$ with length one $\}$.

3. The following proposition is well known (e.g., see Proposition 1 in [Wi-1]); we will use it in the next section. The proof is left to the reader.

(3.1) Proposition. Let $X$ and $Y$ be compact metric spaces and let $\left\{F_{n}\right\}_{n=1}^{\infty}$ and $\left\{K_{n}\right\}_{n=1}^{\infty}$ be two sequences of finite collections of compact sets satisfying:

(3.1.1) For each $n, K_{n}^{*}=Y$ and $F_{n}^{*}=X$; and $\lim _{n \rightarrow \infty} \mu\left(K_{n}\right)=0$.

(3.1.2) There exists a one-to-one and onto function $T_{n}: F_{n} \rightarrow K_{n}$ such that:

(a) For $n \geqslant 2$, if $f_{n} \in F_{n}, f_{n-1} \in F_{n-1}$ with $f_{n} \subseteq f_{n-1}$, then $T_{n}\left(f_{n}\right) \subseteq$ $T_{n-1}\left(f_{n-1}\right)$.

(b) If $x \in X$, then there is a sequence $\left\{f_{n}\right\}_{n=1}^{\infty}$ with $x \in f_{n} \in F_{n}$ and with, for $n \geqslant 2, f_{n} \subseteq f_{n-1}$.

(c) If $y \in Y$, then there is a sequence $\left\{k_{n}\right\}_{n=1}^{\infty}$ with $y \in k_{n} \in K_{n}$ and with, for $n>2, k_{n} \subseteq k_{n-1}$ and $T_{n}^{-1}\left(k_{n}\right) \subseteq T_{n-1}^{-1}\left(k_{n-1}\right)$. 
(3.1.3) If $f_{n}^{1}, \ldots, f_{n}^{q} \in F_{n}$ and $f_{n}^{1} \cap \cdots \cap f_{n}^{q} \neq \varnothing$, then $T_{n}\left(f_{n}^{1}\right) \cap \cdots \cap$ $T_{n}\left(f_{n}^{q}\right) \neq \varnothing$. Then there exists a mapping $g$ from $X$ onto $Y$ defined by

$$
g\left(\bigcap_{n=1}^{\infty} f_{n}\right)=\bigcap_{n=1}^{\infty} T_{n}\left(f_{n}\right)
$$

for each nested sequence $\left\{f_{n}\right\}_{n=1}^{\infty}$ with $f_{n} \in F_{n}$.

4. The following theorem is a modest version of the Main Theorem; the proof of Theorem (4.1) is based on Proposition (4.2) and is presented at the end of this section.

(4.1) THEOREM. Assume the hypothesis of the Main Theorem. Then there is an isotopy $\left\{h_{t}\right\}_{t \in[0, \infty)}$ and a mapping $g=\lim _{t \rightarrow \infty}\left(f \circ h_{t}\right)$ satisfying all the conclusions of the Main Theorem except that, in place of $g$ being open, we have that $g$ is admissible.

(4.2) Proposition. Let $M^{m}$ be a compact, connected manifold with $m>3$, let $f$ be a monotone mapping of $M$ onto $Y$, and let $\varepsilon>0$. For each positive integer $n$, there is a monotone mapping $H_{n}$ from $M$ onto $Y$ and a $H_{n}$-admissible closed brick partition $J_{n}$ of $Y$ satisfying:

(4.2.1) $\mu\left(J_{1}\right)<\varepsilon / 3$ and, for $n \geqslant 2, J_{n}$ refines $J_{n-1}$ and $\mu\left(J_{n}\right)<\varepsilon_{n} / 2^{n}$ where

$$
\begin{aligned}
\xi_{n}=\min \left\{\xi_{2}, \ldots, \xi_{n-1},\right. & \\
& \left.\min \left\{d\left(j_{n-1},\left(J_{n-1}-\operatorname{st}\left(j_{n-1}, J_{n-1}\right)\right)\right)^{*} \mid j_{n-1} \in J_{n-1}\right\}\right\} .
\end{aligned}
$$

(4.2.2) For $n \geqslant 2$, if $j_{n} \in J_{n}$ and $j_{n-1} \in J_{n-1}$ with $j_{n} \subseteq j_{n-1}$, then

$$
H_{n}^{-1}\left(\operatorname{int}\left(j_{n}\right)\right) \subseteq H_{n-1}^{-1}\left(\operatorname{int}\left(j_{n-1}\right)\right) \subseteq N_{1 / 2^{2}}\left(H_{n}^{-1}\left(\operatorname{int}\left(j_{n}\right)\right)\right) .
$$

Proof. Let $\left\{\mathcal{G}_{i}\right\}_{i=1}^{\infty}$ be a sequence of $f$-admissible closed brick partitions of $Y$ with $\mathcal{G}_{i}$ refining $\mathcal{G}_{i-1}$ and with $\lim _{i \rightarrow \infty} \mu\left(\mathcal{S}_{i}\right)=0$. Choose $i_{1}$ such that $\mu\left(\mathcal{G}_{i_{1}}\right)<\varepsilon / 3$; let $J_{1}=\mathcal{G}_{i_{1}}$ and $H_{1}=f$. We will now present the construction of the $n=2$ stage; the method for going from the $n$th stage to the $(n+1)$ st stage is essentially the same construction.

Choose $i_{2}>i_{1}$ such that $\mu\left(\mathcal{G}_{i_{2}}\right)<\xi_{2} / 2^{2}$ and let $J_{2}=\mathcal{G}_{i_{2}}$. Let $\Gamma=\left\{\left(j_{2}, j_{1}\right)\right.$ $\left.\in J_{2} \times J_{1} \mid j_{2} \subseteq j_{1}\right\}$. For each $\gamma=\left(j_{2}, j_{1}\right) \in \Gamma$, let $\beta_{\gamma} \subseteq H_{1}^{-1}\left(\operatorname{int}\left(j_{1}\right)\right)$ be an arc with $\beta_{\gamma}(0) \in H_{1}^{-1}\left(\operatorname{int}\left(j_{2}\right)\right)$ and with $H_{1}^{-1}\left(\operatorname{int}\left(j_{1}\right)\right) \subseteq N_{1 / 2^{2}}\left(\beta_{\gamma}\right)$; and let $U_{\gamma} \subseteq H_{1}^{-1}\left(\operatorname{int}\left(j_{1}\right)\right)$ be an open neighborhood of $\beta_{\gamma}$. We can assume that the $U_{\gamma}$ 's are pairwise disjoint. Let $\left\{k_{t}^{2}\right\}_{t \in[0,1]}$ be an isotopy of $M$ with $k_{0}^{2}=$ identity, with $\operatorname{supp}\left(\left\{k_{t}^{2}\right\}_{t \in[0,1]} \subseteq\left\{U_{\gamma} \mid \gamma \in \Gamma\right\}^{*}\right.$, and with $\beta_{\gamma} \subseteq$ $\left(H_{1} \circ k_{1}\right)^{-1}\left(\operatorname{int}\left(j_{2}\right)\right)$ for each $\gamma=\left(j_{2}, j_{1}\right) \in \Gamma$.

(4.3) REMARK. We need to be sure that each of the $\beta_{\gamma}$ 's is chosen so that any neighborhood of $\beta_{\gamma}(0)$ can be "pulled over" the entire arc $\beta_{\gamma}$. There is no difficulty in making such choices; henceforth, we will implicitly assume that all arcs used are so chosen. 
The $n=2$ stage is completed by letting $H_{2}=H_{1} \circ k_{1}^{2}$. The inductive step is done similarly by choosing $i_{n}>i_{n-1}$ such that $\mu\left(\mathcal{G}_{i_{n}}\right)<\xi_{n} / 2^{n}$ and letting $J_{n}=\mathcal{G}_{i_{n}}$ and $\Gamma=\left\{\left(j_{n}, j_{n-1}\right) \in J_{n} \times J_{n-1} \mid j_{n} \subseteq j_{n-1}\right\}$, and using $H_{n-1}$ in place of $H_{1}$ and $N_{1 / 2^{n}}\left(\beta_{\gamma}\right)$ in place of $N_{1 / 2^{2}}\left(\beta_{\gamma}\right)$.

Proof of Theorem (4.1). (4.4) Define the isotopy $\left\{h_{t}\right\}_{t \in[0, \infty)}$ as follows. Let $h_{t}=$ identity for $t \in[0,1]$ and, for each integer $n \geqslant 1$, if $t \in[n, n+1]$, then let $h_{t}=k_{1}^{2} \circ k_{1}^{3} \circ \cdots \circ k_{1}^{n} \circ k_{t-n}^{n+1}$. Observe that $H_{n}=f \circ h_{n}$. We will now verify that $\lim _{n \rightarrow \infty} H_{n}$ exists; we leave to the reader to verify that $\lim _{t \rightarrow \infty}\left(f \circ h_{t}\right)$ exists.

Let $K_{n}=\left\{j_{n}^{1} \cup \cdots \cup j_{n}^{q} \mid j_{n}^{1} \cap \cdots \cap j_{n}^{q} \neq \varnothing\right.$ and $j_{n} \cap\left(j_{n}^{1} \cap \cdots \cap j_{n}^{q}\right)$ $=\varnothing$ for each $\left.j_{n} \in J_{n}-\left\{j_{n}^{1}, \ldots, j_{n}^{q}\right\}\right\}$. Let $F_{n}=\left\{\operatorname{cl}\left(H_{n}^{-1}\left(\operatorname{int}\left(k_{n}\right)\right)\right) \mid k_{n} \in K_{n}\right\}$ and let $T_{n}\left(\operatorname{cl}\left(H_{n}^{-1}\left(\operatorname{int}\left(k_{n}\right)\right)\right)\right)=k_{n}$. It is easily verified that the sequence of triples $T_{n}: F_{n} \rightarrow K_{n}$ satisfies the hypothesis of Proposition (3.1); it is useful to have the observation that, since $J_{n}$ is $H_{n}$-admissible, if $k_{n}=j_{n}^{1} \cup \cdots \cup j_{n}^{q} \in$ $K_{n}$, then

$$
\operatorname{cl}\left(H_{n}^{-1}\left(\operatorname{int}\left(k_{n}\right)\right)\right)=\operatorname{cl}\left(H_{n}^{-1}\left(\operatorname{int}\left(j_{n}^{1}\right)\right)\right) \cup \cdots \cup \operatorname{cl}\left(H_{n}^{-1}\left(\operatorname{int}\left(j_{n}^{q}\right)\right)\right) .
$$

Let $g$ be the mapping defined in (3.1); we now check that $\lim _{n \rightarrow \infty} H_{n}=g$. Let $\delta>0$ and let $n_{0}$ be such that $\mu\left(K_{n}\right)<\delta$ for $n \geqslant n_{0}$. Let $x \in M$ and let $\left\{f_{n}\right\}_{n=1}^{\infty}$ be a nested sequence with $f_{n} \in F_{n}$ and $x \in \bigcap_{n=1}^{\infty} f_{n}$. For each $n$, $H_{n}(x) \in T_{n}\left(f_{n}\right)$ and, since $g(x)=\bigcap_{n=1}^{\infty} T_{n}\left(f_{n}\right), g(x) \in T_{n}\left(f_{n}\right)$. Therefore, if $n \geqslant n_{0}$, then $d\left(g(x), H_{n}(x)\right)<\delta$.

We now show that $g$ is admissible. Let $L$ be a closed subset of $Y$ with $\operatorname{int}(L)=\varnothing$. Let $x \in g^{-1}(L)$ and let $\eta>0$; we will now produce $x^{\prime} \in N_{\eta}(x)$ with $g\left(x^{\prime}\right) \notin L$. Let $n_{0}$ be such that $\sum_{n \geqslant n_{0}} 1 / 2^{n}<\eta$. Let $j_{n_{0}} \in J_{n_{0}}$ with $N_{1 / 2^{n_{0}}}(x) \cap H_{n_{0}}^{-1}\left(\operatorname{int}\left(j_{n_{0}}\right)\right) \neq \varnothing$ and let $n_{1}>n_{0}$ be such that there is a $j_{n_{1}} \in J_{n_{1}}$ with $j_{n_{1}} \subseteq j_{n_{0}}$ and $\operatorname{st}\left(j_{n_{1}}, J_{n_{1}}\right) * L=\varnothing$. Let $j_{n_{0}}, j_{n_{0}+1}, \ldots, j_{n_{1}}$ be a nested sequence with $j_{n_{0}+i} \in J_{n_{0}+i}$ for $i=1, \ldots, n_{1}-n_{0}$. Let $x_{n_{0}} \in N_{1 / 2^{n_{0}}}(x) \cap$ $H_{n_{0}}^{-1}\left(\operatorname{int}\left(j_{n_{0}}\right)\right)$ and, inductively for $i=n_{0}+1, \ldots, n_{1}$, recalling condition (4.2.2), let $x_{i} \in N_{1 / 2^{i}}\left(x_{i-1}\right) \cap H_{i}^{-1}\left(\operatorname{int}\left(j_{i}\right)\right)$. Observe that $d\left(x, x_{n_{1}}\right)<$ $\sum_{i=n_{0}}^{n_{1}} 1 / 2^{i}<\eta$ and it is easy to verify that $g\left(x_{n_{1}}\right) \in \operatorname{st}\left(j_{n_{1}}, J_{n_{1}}\right)^{*}$; let $x^{\prime}=x_{n_{1}}$.

To be sure that each $g^{-1}(y)$ is nondegenerate, we must change the construction in the proof of Proposition (4.2) as follows. For each $j_{1} \in J_{1}$, let $B_{j_{1}}^{1}$ and $B_{j_{1}}^{2}$ be disjoint nonempty, open subsets of $H_{1}^{-1}\left(\operatorname{int}\left(j_{1}\right)\right)$. A more careful choice of the $\beta_{\gamma}$ 's will insure that for each $n \geqslant 1$, if $j_{n} \in J_{n}$ and $j_{n} \subseteq j_{1} \in J_{1}$, then $H_{n}^{-1}\left(\operatorname{int}\left(j_{n}\right)\right) \cap B_{j_{1}}^{i} \neq \varnothing, i=1,2$. We leave to the reader to verify that this modification guarantees that each $g^{-1}(y)$ is nondegenerate.

5. Proposition (5.1) below is an embellished version of Proposition (3.1) and is used to produce open mappings ((5.1) appears as Proposition 2 in Wilson's paper [Wi-1] and the reader is referred there for a proof). Proposition (5.2) is 
the technical device used to govern the construction contained in the next section; (5.2) is a variation of Wilson's Proposition 3 in [Wi-1]. Both these propositions have their genesis in Anderson's work [A-2], [A-3], [A-4].

(5.1) Proposition. Assume in addition to the hypothesis of Proposition (3.1) that:

(5.1.1) If $k_{n}, k_{n}^{\prime} \in K_{n}$ and $k_{n} \cap k_{n}^{\prime} \neq \varnothing$, then

$$
T_{n}^{-1}\left(k_{n}\right) \cap T_{n}^{-1}\left(k_{n}^{\prime}\right) \neq \varnothing .
$$

(5.1.2) There is $a>0$ such that if $f_{n}, f_{n}^{\prime} \in F_{n}$ and $f_{n} \cap f_{n}^{\prime} \neq \varnothing$, then

$$
f_{n} \subseteq N_{a / 2^{n}}\left(f_{n}^{\prime}\right) .
$$

(5.1.3) There is $b>0$ such that if $f_{n} \in F_{n}$ and $f_{n-1} \in F_{n-1}$ with $f_{n} \subseteq f_{n-1}$, then

$$
f_{n-1} \subseteq N_{b / 2^{n}}\left(f_{n}\right) .
$$

Then the mapping $g$ constructed will be open.

(5.2) Proposition. Let $X$ and $Y$ be compact metric spaces and let $\left\{J_{n}\right\}_{n=1}^{\infty}$ and $\left\{P_{n}\right\}_{n=1}^{\infty}$ be two sequences of finite collection of compact sets satisfying:

$$
J_{n}^{*}=Y \quad \text { and } P_{n}^{*}=X ; \lim _{n \rightarrow \infty} \mu\left(J_{n}\right)=0 .
$$

(5.2.2) The members of $J_{n}$ (resp., $P_{n}$ ) have pairwise disjoint nonempty interiors; and, for each $j_{n} \in J_{n}, j_{n}=\operatorname{cl}\left(\operatorname{int}\left(j_{n}\right)\right)$.

(5.2.3) There is a one-to-one and onto function $R_{n}: J_{n} \rightarrow P_{n}$ such that:

(i) if $p_{n}^{1}, \ldots, p_{n}^{q} \in P_{n}$ and $p_{n}^{1} \cap \cdots \cap p_{n}^{q} \neq \varnothing$, then $R_{n}^{-1}\left(p_{n}^{1}\right) \cap \cdots \cap$ $R_{n}^{-1}\left(p_{n}^{q}\right) \neq \varnothing$;

(ii) if $j_{n}, j_{n}^{\prime} \in J_{n}$ and $j_{n} \cap j_{n}^{\prime} \neq \varnothing$, then $R_{n}\left(j_{n}\right) \cap R_{n}\left(j_{n}^{\prime}\right) \neq \varnothing$.

(5.2.4) For $n \geqslant 2$, if $j_{n} \in J_{n}$ and $j_{n-1} \in J_{n-1}$ with $j_{n} \cap \operatorname{int}\left(j_{n-1}\right) \neq \varnothing$, then $\operatorname{int}\left(R_{n}\left(j_{n}\right)\right) \cap \operatorname{int}\left(R_{n-1}\left(j_{n-1}\right)\right) \neq \varnothing$.

(5.2.5) For $n \geqslant 2$, if $j_{n} \in J_{n}$, then $\cap\left\{j_{n-1} \in J_{n-1} \mid R_{n-1}\left(j_{n-1}\right) \cap R_{n}\left(j_{n}^{\prime}\right) \neq \varnothing\right.$ for some $\left.j_{n}^{\prime} \in \operatorname{st}\left(j_{n}, J_{n}\right)\right\} \neq \varnothing$.

(5.2.6) There is $c>0$ such that if $j_{n}, j_{n}^{\prime} \in J_{n}$ and $j_{n} \cap j_{n}^{\prime} \neq \varnothing$, then $R_{n}\left(j_{n}\right) \subseteq$ $N_{c / 2^{n}}\left(R_{n}\left(j_{n}^{\prime}\right)\right)$.

(5.2.7) There is a $d>0$ such that, for $n \geqslant 2$, if $j_{n} \in J_{n}$ and $j_{n-1} \in J_{n-1}$ with $j_{n} \cap \operatorname{int}\left(j_{n-1}\right) \neq \varnothing$, then $R_{n-1}\left(j_{n-1}\right) \subseteq N_{d / 2^{n}}\left(R_{n}\left(j_{n}\right)\right)$.

Then, letting $K_{n}=\left\{j_{n}^{1} \cup \cdots \cup j_{n}^{q} \mid j_{n}^{1} \cap \cdots \cap j_{n}^{q} \neq \varnothing\right.$ and $j_{n} \cap\left(j_{n}^{1}\right.$ $\left.\cap \cdots \cap j_{n}^{q}\right)=\varnothing$ for $\left.j_{n} \in J_{n}-\left\{j_{n}^{1}, \ldots, j_{n}^{q}\right\}\right\}$, letting $F_{n}=\left\{R_{n}\left(j_{n}^{1}\right)\right.$ $\left.\cup \cdots \cup R_{n}\left(j_{n}^{q}\right) \mid j_{n}^{1} \cup \cdots \cup j_{n}^{q} \in K_{n}\right\}$ and letting $T_{n}\left(R_{n}\left(j_{n}^{1}\right) \cup \cdots \cup\right.$ $\left.R_{n}\left(j_{n}^{q}\right)\right)=j_{n}^{1} \cup \cdots \cup j_{n}^{q}$, the triples $T_{n}: F_{n} \rightarrow K_{n}$ satisfy the hypothesis of Proposition (5.1) with $a=2 c$ and $b=4 c+d$.

Proof. It is easy to show that conditions (3.1.1), (3.1.3), and (5.1.1) hold and conditions (5.1.2) and (5.1.3) are direct consequences of (5.2.6) and (5.2.7). 
Condition (3.1.2). (a) Let $f_{n} \in F_{n}$ and $f_{n-1} \in F_{n-1}$ with $f_{n} \subseteq f_{n-1}$. Suppose that $T_{n}\left(f_{n}\right) \underline{Z} T_{n-1}\left(f_{n-1}\right)$; then there is $j_{n} \subseteq T_{n}\left(f_{n}\right)$ with $j_{n} \& T_{n-1}\left(f_{n-1}\right)$ and $j_{n-1} \& T_{n-1}\left(f_{n-1}\right)$ with $j_{n} \cap \operatorname{int}\left(j_{n-1}\right) \neq \varnothing$. Property (5.2.4) implies that $f_{n} \cap$ $\operatorname{int}\left(R_{n-1}\left(j_{n-1}\right)\right) \neq \varnothing$; since $\operatorname{int}\left(R_{n-1}\left(j_{n-1}\right)\right) \cap f_{n-1}=\varnothing$, we have the contradiction that $f_{n} \& f_{n-1}$. Hence we must have $T_{n}\left(f_{n}\right) \subseteq T_{n-1}\left(f_{n-1}\right)$.

Condition (3.1.2). (b) Let $x \in X$ and let $j_{n}^{x} \in J_{n}$ with $x \in R_{n}\left(j_{n}^{x}\right)$. Let $D_{n-1}^{x}=\left\{j_{n-1} \in J_{n-1} \mid\right.$ there is a $j_{n} \in \operatorname{st}\left(j_{n}^{x}, J_{n}\right)$ with $R_{n-1}\left(j_{n-1}\right) \cap R_{n}\left(j_{n}\right)$ $\neq \varnothing\}$. Property (5.2.5) implies that there is $k_{n-1}^{x} \in K_{n-1}$ with $\left(D_{n-1}^{x}\right)^{*} \subseteq k_{n-1}^{x}$. If $x \in R_{n-1}\left(j_{n-1}\right)$, then property (5.2.4) implies that $j_{n-1} \in D_{n-1}^{x}$; hence, $j_{n-1}^{x} \subseteq k_{n-1}^{x}$ and $x \in T_{n-1}^{-1}\left(k_{n-1}^{x}\right)$. Letting $f_{n-1}^{x}=T_{n-1}^{-1}\left(k_{n-1}^{x}\right)$, we will now show that a sequence $\left\{f_{n}^{x}\right\}$ chosen as above is nested. Since $T_{n}\left(f_{n}^{x}\right) \subset$ $\operatorname{st}\left(j_{n}^{x}, J_{n}\right)^{*}$, it suffices to show that $R_{n}\left(j_{n}\right) \subseteq f_{n-1}^{x}$ for each $j_{n} \in \operatorname{st}\left(j_{n}^{x}, J_{n}\right)$. To this end, notice that if $j_{n} \in \operatorname{st}\left(j_{n}^{x}, J_{n}\right)$ and $R_{n}\left(j_{n}\right) \cap R_{n-1}\left(j_{n-1}\right) \neq \varnothing$, then $j_{n-1} \in D_{n-1}^{x}$; therefore, $R_{n}\left(j_{n}\right) \subseteq f_{n-1}^{x}$.

Condition (3.1.2). (c) Let $y \in Y$ and let $j_{n}^{y} \in J_{n}$ with $y \in j_{n}^{y}$. Let $E_{n-1}^{y}=$ $\left\{j_{n-1} \in J_{n-1} \mid\right.$ there is a $j_{n} \in \operatorname{st}\left(j_{n}^{y}, J_{n}\right)$ with $\left.R_{n-1}\left(j_{n-1}\right) \cap R_{n}\left(j_{n}\right) \neq \varnothing\right\}$; let $k_{n-1}^{y} \in K_{n-1}$ with $\left(E_{n-1}^{y}\right)^{*} \subseteq k_{n-1}^{y}$ (property (5.2.5) guarantees that $k_{n-1}^{y}$ exists). We leave to the reader to use (5.2.5) to verify that a sequence $\left\{k_{n}^{y}\right\}$ chosen as above is nested and that $T_{n}^{-1}\left(k_{n}^{y}\right) \subseteq T_{n-1}^{-1}\left(k_{n-1}^{y}\right)$.

6. Proposition (6.1) below together with Propositions (5.1) and (5.2) will be used to prove the Main Theorem in much the same manner that Propositions (4.2) and (3.1) were used to prove Theorem (4.1).

(6.1) Proposition. Let $M^{m}$ be a compact, connected manifold with $m>3$, let $f$ be a monotone mapping of $M$ onto $Y$, and let $\varepsilon>0$. For each positive integer $n$, there is a monotone mapping $H_{n}$ from $M$ onto $Y$ and $a H_{n}$-admissible closed brick partition $J_{n}$ of $Y$ satisfying:

(6.1.1) $\mu\left(J_{1}\right)<\varepsilon / 3$ and, for $n \geqslant 2, J_{n}$ refines $J_{n-1}$ and $\mu\left(J_{n}\right)<\xi_{n} / 2^{n}$ where $\xi_{n}=\min \left\{\xi_{2}, \ldots, \xi_{n-1}, \min \left\{d\left(j_{n-1},\left(Y-\operatorname{st}\left(j_{n-1}, J_{n-1}\right)^{*}\right)\right) \mid j_{n-1} \in J_{n-1}\right\}\right\}$.

(6.1.2) For $n \geqslant 2$, if $j_{n} \in J_{n}$ and $j_{n-1} \in J_{n-1}$ with $j_{n} \cap j_{n-1} \neq \varnothing$, then $H_{n}^{-1}\left(\operatorname{int}\left(j_{n}\right)\right) \cap H_{n-1}^{-1}\left(\operatorname{int}\left(j_{n-1}\right)\right) \neq \varnothing$.

(6.1.3) For $n \geqslant 2$, if $j_{n} \in J_{n}$, then $\cap\left\{j_{n-1} \in J_{n-1} \mid \operatorname{cl}\left(H_{n-1}^{-1}\left(\operatorname{int}\left(j_{n-1}\right)\right)\right) \cap\right.$ $\operatorname{cl}\left(H_{n}^{-1}\left(\operatorname{int}\left(j_{n}^{\prime}\right)\right)\right) \neq \varnothing$ for some $\left.j_{n}^{\prime} \in \operatorname{st}\left(j_{n}, J_{n}\right)\right\} \neq \varnothing$.

(6.1.4) If $j_{n}, j_{n}^{\prime} \in J_{n}$ with $j_{n} \cap j_{n}^{\prime} \neq \varnothing$, then $H_{n}^{-1}\left(\operatorname{int}\left(j_{n}\right)\right) \subseteq$ $N_{1 / 2^{n}}\left(H_{n}^{-1}\left(\operatorname{int}\left(j_{n}^{\prime}\right)\right)\right)$.

(6.1.5) For $n \geqslant 2$, if $j_{n} \in J_{n}$ and $j_{n-1} \in J_{n-1}$ with $j_{n} \cap j_{n-1} \neq \varnothing$, then $H_{n-1}^{-1}\left(\operatorname{int}\left(j_{n-1}\right)\right) N_{1 / 2^{n}}\left(H_{n}^{-1}\left(\operatorname{int}\left(j_{n}\right)\right)\right)$.

Discussion. Although the proof of Proposition (6.1) is long, the mapping $H_{n}$ is obtained from $H_{n-1}$ by a finite sequence of alterations with each individual alteration consisting of "pulling" a small neighborhood of one endpoint of an arc in $M$ over the entire arc and "alongside" several other 
arcs. The proof of Proposition (6.1) splits into three basic parts; first, in (6.2)-(6.4), we construct the partition $J_{n}$ and develop a "bookkeeping system" (which is unfortunately very complicated) which contains a detailed description of each individual description of each individual alteration; second, in (6.5) we give a global description of the alterations used to obtain $H_{n}$ from $H_{n-1}$ (it is difficult to prove that $H_{n}$ satisfies condition (6.1.4) ${ }^{n}$ using the global description); in (6.6), we obtain $H_{n}$ from $H_{n-1}$ by a sequence of alterations each of which is part of an alteration described in (6.5) and, simultaneously, we prove that $H_{n}$ satisfies condition (6.1.4) .

The remainder of the discussion is devoted to: first, a comparison of Propositions (6.1) and (4.2) and a description of the central difficulty encountered in proving (6.1); second, a comparison of the approach used to prove Proposition (6.1) with those used in [Wa-1], [Wa-2] and [Wi-1], [Wi-2]; third, an outline of the proof of Proposition (6.1) for a special case and suggestions which should be helpful in reading the proof of Proposition (6.1).

A COMParison of Propositions (4.2) AND (6.1). Conditions (6.1.1) and (4.2.1) are exactly the same; part of condition (4.2.2) is retained in condition (6.1.5). In Proposition (4.2), if $j_{n} \subseteq j_{n-1}$, then $H_{n}^{-1}\left(\operatorname{int}\left(j_{n}\right)\right) \subseteq H_{n-1}^{-1}\left(\operatorname{int}\left(j_{n-1}\right)\right)$ and condition (6.1.2) necessarily held; although it is necessary to state condition (6.1.2) explicitly, the construction is such that it is easily seen to hold. Conditions (6.1.3) and (6.1.4) represent the essential differences between Propositions (6.1) and (4.2). Condition (6.1.3) is needed to insure that the $H_{n}$ 's converge to a function (the information needed is that if $H_{n}^{-1}\left(j_{n}\right) \cap$ $H_{n-1}^{-1}\left(j_{n-1}\right) \neq \varnothing$, then $j_{n}$ is "close to" $\left.j_{n-1}\right)$. Condition (6.1.4) guarantees that the $H_{n}$ 's converge to an open mapping.

The major difficulty in the proof of Proposition (6.1) is to achieve condition (6.1.4) subject to the constraint imposed by condition (6.1.3). Condition (6.1.4) is extremely "delicate" since each time $H_{n-1}^{-1}\left(j_{n}\right)$ is altered it is necessary to alter $H_{n-1}^{-1}\left(j_{n}^{\prime}\right)$ for each $j_{n}^{\prime} \in \operatorname{st}\left(j_{n}, J_{n}\right)$; this makes it necessary to alter $H_{n}^{-1}\left(j_{n}^{\prime \prime}\right)$ for each $j_{n}^{\prime \prime} \in \operatorname{st}\left(j_{n}^{\prime}, J_{n}\right)$ where $j_{n}^{\prime} \in \operatorname{st}\left(j_{n}, J_{n}\right) \ldots$ The "bookkeeping system" established in (6.3) lists each alteration which is to be made; the complexity of the system results from the interdependence of various alterations.

A comparison of Proposition (6.1) and Proposition (3.1) in [Wa-1]. Those readers not familiar with [Wa-1] may skip this paragraph; in this paragraph only (3.1.•) will refer to conditions in Proposition (3.1) in [Wa-1]. The role of $K_{n}$ 's and $R_{n}$ 's in Proposition (3.1) is played by the $H_{n}$ 's (i.e., we define $K_{n}$ and $R_{n}$ by letting $K_{n}=\left\{\operatorname{cl}\left(H_{n}^{-1}\left(\operatorname{int}\left(j_{n}\right)\right)\right) \mid j_{n} \in J_{n}\right\}$ and letting $R_{n}\left(\operatorname{cl}\left(H_{n}^{-1}\left(\operatorname{int}\left(j_{n}\right)\right)\right)\right)$ $=j_{n}$ ). The first part of condition (3.1.3) is automatically satisfied and the second part is essentially condition (6.1.2). Conditions (3.1.2) and (3.1.4) are replaced by condition (6.1.3); condition (3.1.6) is replaced by condition 
(6.1.5); and, condition (3.1.5) is replaced by condition (6.1.4). Notice that there is not a condition in Proposition (6.1) which plays the role of condition (3.1.7); the latter condition and the rule in (3.3) are used to achieve condition (3.1.5). There is a definite temptation to use a variation of (3.1.7) and (3.3) in the proof of Proposition (6.1) in order to achieve condition (6.1.4); and such an approach can be used to get condition (6.1.4) partially satisfied. This author's experience with several such approaches convinced him that if a method can be used to achieve condition (6.1.4) exactly, then the method can be used from the outset; i.e., without partially achieving condition (6.1.4) by the preliminary use of a variation of (3.1.7) and (3.3). (In fact, there is a precise sense in which the method used in proving Proposition (3.1) is a "nonisotopic" method.) Nevertheless, those familiar with [Wa-1], [Wa-2] and [Wi-1] can benefit by contemplating an approach to the proof of Proposition (6.1) based on the technique used therein.

AN outline of THE PROOF OF Proposition (6.1). Suppose that $J_{n-1}$ and $H_{n-1}$ have been constructed; since the information in $(6.1 .1)^{n-1}-(6.1 .5)^{n-1}$ is not needed in order to construct $H_{n}$ and $J_{n}$, it is not necessary to know the method which was used to construct $J_{n-1}$ and $H_{n-1}$. Let $\mathcal{Q}, \mathfrak{K}_{1}, \ldots, \mathfrak{M}_{I(\mathscr{Q})}$ be the filtration of $Y$ obtained from the partition $J_{n-1}$ (see §2) and let $M_{i}=\left\{|x| \mid x \in \mathfrak{M}_{i}\right\}^{*}, i=1,2, \ldots, I(\mathbb{Q})$. The partition $J_{n}$ will be a union, $J_{n}^{1} \cup \cdots \cup J_{n}^{I(\mathcal{Q})}$, of pairwise disjoint collections with

$$
M_{1} \cup \cdots \cup M_{i} \subseteq \operatorname{int}\left(\left(J_{n}^{1} \cup \cdots \cup J_{n}^{i}\right)^{*}\right) \text { for } i=1,2, \ldots, I(\mathbb{Q}) .
$$

For the remainder of the discussion, we are going to assume that $Y$ is a 2 dimensional manifold (for convenience, without boundary) with the bricks in the partitions being 2-cells which intersect in arcs. In particular, $I(\mathbb{Q})=3, M_{1}$ is a collection of points, $M_{2}$ is a collection of 1-cells, and $M_{3}$ is a collection of 2-cells. (The only special property of 2-manifolds we want to use is that $I(\mathbb{Q})=3$; the case with $I(\mathscr{Q})=3$ is sufficiently complex in order to illustrate the approach which is used to achieve condition (6.1.4).) Recall that $J_{n}$ will be the union of collections $J_{n}^{1}, J_{n}^{2}$, and $J_{n}^{3} ; J_{n}^{1}$ consists of bricks which intersect $M_{1}, J_{n}^{2}$ consists of bricks which intersect $M_{2}-\left(J_{n}^{1}\right)^{*}$, and $J_{n}^{3}$ consists of bricks which intersect $M_{3}-\left(J_{n}^{1} \cup J_{n}^{2}\right)^{*}$; see Figure 1. In Figure 1, notice that the bricks in $J_{n}^{2}$ (resp., $J_{n}^{3}$ ) are smaller than those in $J_{n}^{1}$ (resp., $J_{n}^{2}$ ); in fact, the actual difference in size is greater than the difference which is illustrated.

The collections $J_{n}^{1}, J_{n}^{2}$, and $J_{n}^{3}$ are constructed inductively as follows.

Step 1. The collection $J_{n}^{1}$ is chosen as is indicated in Figure 1 with $M_{1} \subseteq \operatorname{int}\left(\left(J_{n}^{1}\right)^{*}\right)$; note that each element of $J_{n}^{1}$ meets $M_{1}$; in addition, the diameter of the elements of $J_{n}^{1}$ should be "small". For each pair $j_{n} \in J_{n}^{1}$ and $j_{n-1} \in J_{n-1}$ with $j_{n} \cap j_{n-1} \neq \varnothing$, a $m$-ball $Q \subseteq H_{n-1}^{-1}\left(\operatorname{int}\left(j_{n}\right)\right)$ is chosen with diameter less than $1 / 2^{n+2}$ and an arc $\eta \subseteq H_{n-1}^{-1}\left(\operatorname{int}\left(j_{n} \cup j_{n-1}\right)\right)$ is chosen with one endpoint in $Q$ and with $H_{n-1}^{-1}\left(\operatorname{int}\left(j_{n-1}\right)\right)$ contained in the $1 / 2^{n+1}$ neigh- 
borhood of $\eta$. One alteration of $H_{n-1}$ is to "pull" a small neighborhood of the endpoint of $\eta$ in $Q$ over the entire arc $\eta$. Each such arc is divided into subarcs whose diameters are less than $1 / 2^{n+2}$. Let $E_{1}$ be the maximum number of subarcs needed for any such arc and let $\mathcal{E}_{1}=E_{1}+1$. Throughout the construction the arcs (resp., $m$-balls) chosen should be pairwise disjoint; and the only intersections of arcs and $m$-balls are those specified.

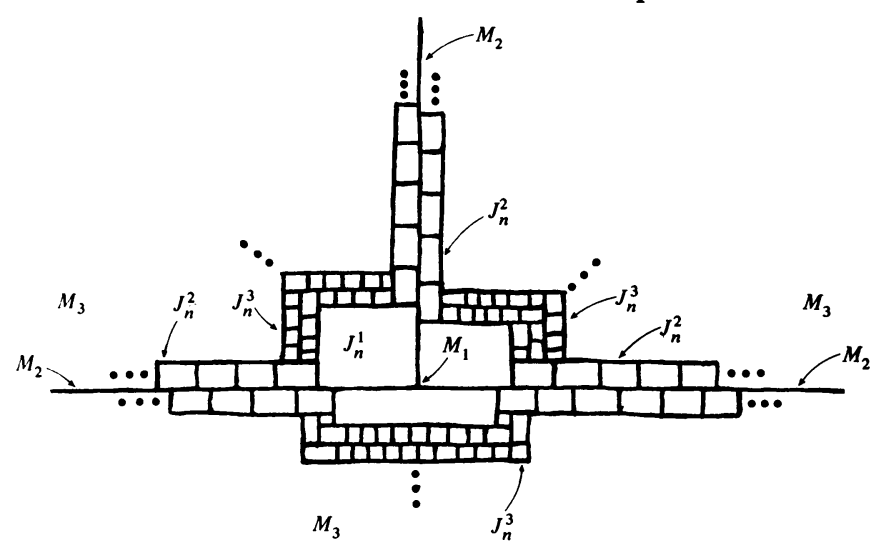

Figure 1

Step 2. The collection $J_{n}^{2}$ is chosen as is indicated in Figure 1 with $M_{2} \subseteq \operatorname{int}\left(\left(J_{n}^{1} \cup J_{n}^{2}\right)^{*}\right)$, with each element of $J_{n}^{2}$ meeting $M_{2}$, and such that if $j_{n} \in J_{n}^{1}$ and $\left(j_{n}^{1}, \ldots, j_{n}^{\delta_{1}}\right)$ is a chain of elements from $J_{n}^{2}$ with $j_{n} \cap j_{n}^{1} \neq \varnothing$, then $j_{n}^{1} \cup \cdots \cup j_{n}^{\mathcal{E}_{1}}$ is "close to" $j_{n}$. For each pair $j_{n} \in J_{n}^{2}$ and $j_{n-1} \in J_{n-1}$ with $j_{n} \cap j_{n-1} \neq \varnothing$, an $m$-ball $Q$ and an arc $\eta$ are chosen as in Step 1; for each such $Q$ and $\eta$ an alteration as described in Step 1 will be done. For each $j_{n} \in J_{n}^{1}$, each $m$-ball $Q \subseteq H_{n-1}^{-1}\left(\operatorname{int}\left(j_{n}\right)\right)$ previously chosen, and each chain $\left(j_{n}^{1}, \ldots, j_{n}^{\mathcal{E}_{1}}\right)$ of elements from $J_{n}^{2}$ with $j_{n} \cap j_{n}^{1} \neq \varnothing$ do the following: choose arcs $\alpha_{1}, \ldots, \alpha_{\mathscr{E}_{1}}$ and $m$-balls $Q_{1}, \ldots, Q_{\mathscr{E}_{1}}$ (the $m$-balls should have diameters less than $1 / 2^{n+2}$ with $Q_{i} \subseteq H_{n-1}^{-1}\left(\operatorname{int}\left(j_{n}^{i}\right)\right)$ for $i=1, \ldots, \mathcal{E}_{1}$, with $\alpha_{1} \subseteq H_{n-1}^{-1}\left(\operatorname{int}\left(j_{n} \cup j_{n}^{1}\right)\right)$ and one endpoint of $\alpha_{1}$ in $Q_{1}$ and the other in $Q$, and with $\alpha_{i} \subseteq H_{n-1}^{-1}\left(\operatorname{int}\left(j_{n}^{i} \cup j_{n}^{i-1}\right)\right)$ and one endpoint of $\alpha_{i}$ in $Q_{i}$ and the other in $Q_{i-1}, i=2, \ldots, \mathcal{E}_{1}$. The $\alpha_{i}$ 's and $Q_{i}$ 's are used to alter $H_{n-1}$ as is indicated in Figure 2; for $i=2, \ldots, \varepsilon_{1}, H_{n-1}^{-1}\left(\operatorname{int}\left(j_{n}^{i}\right)\right)$ is "pulled alongside" one fewer subarc of $\eta$ than $H_{n-1}^{-1}\left(\operatorname{int}\left(j_{n}^{i-1}\right)\right)$ is "pulled alongside". The choice of $\mathcal{E}_{1}$ is such that $H_{n-1}^{-1}\left(\operatorname{int}\left(j_{n}^{\mathcal{E}_{1}}\right)\right)$ is not "pulled alongside" any of $\eta$.

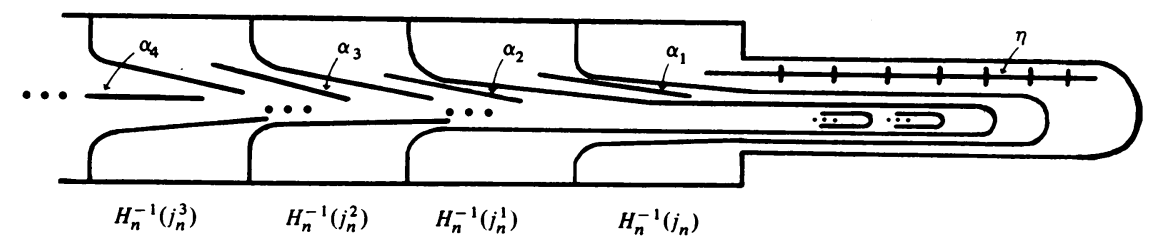

FIGURE 2 
Divide each arc chosen above (both the $\eta$ 's and the $\alpha$ 's) into subarcs whose diameters are less than $1 / 2^{n+2}$. Let $E_{2}$ be the maximum number of subarcs needed for any such arc and let $\varepsilon_{2}=1+\varepsilon_{1}+\varepsilon_{1} \cdot E_{2}$.

Step 3. The collection $J_{n}^{3}$ is chosen as is indicated in Figure 1 with the property that if $j_{n} \in J_{n}^{1} \cup J_{n}^{2}$ and $\left(j_{n}^{1}, \ldots, j_{n}^{\mathcal{E}_{2}}\right)$ is a chain of elements from $J_{n}^{3}$ with $j_{n} \cap j_{n}^{1} \neq \varnothing$, then $j_{n}^{1} \cup \cdots \cup j_{n}^{\mathscr{E}_{2}}$ is "close to" $j_{n}$. For each pair $j_{n} \in J_{n}^{3}$ and $j_{n-1} \in J_{n-1}$ with $j_{n} \cap j_{n-1} \neq \varnothing$, an $m$-ball $Q$ and an arc $\eta$ are chosen as in Step 1; for each such $Q$ and $\eta$, an alteration as described in Step 1 will be done. For each $j_{n} \in J_{n}^{1} \cup J_{n}^{2}$, each $m$-ball $Q \subseteq H_{n-1}^{-1}\left(\operatorname{int}\left(j_{n}\right)\right)$ previously chosen, each chain $\left(j_{n}^{1}, \ldots, j_{n}^{\delta_{2}}\right)$ of elements from $J_{n}^{3}$ with $j_{n} \cap j_{n}^{1} \neq \varnothing$, arcs $\alpha_{1}, \ldots, \alpha_{\mathscr{E}_{2}}$ and $m$-balls $Q_{1}, \ldots, Q_{\varepsilon_{2}}$ are chosen as in Step 2. (Since this is the last step in the construction of $J_{n}=J_{n}^{1} \cup J_{n}^{2} \cup J_{n}^{3}$, it is not necessary to subdivide the arcs chosen above.) Let $j_{n} \in J_{n}^{1} \cup J_{n}^{2}$, let $Q \subseteq H_{n-1}^{-1}\left(\operatorname{int}\left(j_{n}\right)\right)$ be a $m$-ball previously chosen, and let $\left(j_{n}^{1}, \ldots, j_{n}^{\varepsilon_{2}}\right)$ be a chain of elements from $J_{n}^{3}$ with $j_{n} \cap j_{n}^{1} \neq \varnothing$. There are two "types" of $Q$ 's. First, $Q$ was paired with an $\operatorname{arc} \eta$; in this case, an alteration as described in Step 2 will be done. Second, $Q$ was paired with an arc $\hat{\alpha}_{i}$ which was chosen in Step 2 for $\hat{j}_{n}^{1} \in J_{n}^{1}$ and $\left(\hat{j}_{n}, \ldots, \hat{j}_{n}\right)$ a chain of elements from $J_{n}^{2}$ with $\hat{j_{n}} \cap \hat{j}_{n} \neq \varnothing$; in this case, $H_{n-1}$ will be altered as is indicated in Figure 3. Notice that the choice of $\mathcal{E}_{2}$ is such that $H_{n-1}^{-1}\left(\operatorname{int}\left(j_{n}^{\varepsilon_{2}}\right)\right)$ is not "pulled alongside" any of $\hat{\alpha}_{i}$.

The reader is encouraged to "sketch" a proof that condition (6.1.4) ${ }^{n}$ will hold if the alterations indicated above are done (the above outline omits a few technical but important details). The approach to use is to show that if $j_{n} \cap j_{n}^{\prime} \neq \varnothing$ and $j_{n-1} \in J_{n-1}$, then

$$
H_{n}^{-1}\left(\operatorname{int}\left(j_{n}^{\prime}\right)\right) \cap H_{n-1}^{-1}\left(\operatorname{int}\left(j_{n-1}\right)\right) \subseteq N_{1 / 2^{n}}\left(H_{n}^{-1}\left(\operatorname{int}\left(j_{n}\right)\right)\right) \text {. }
$$

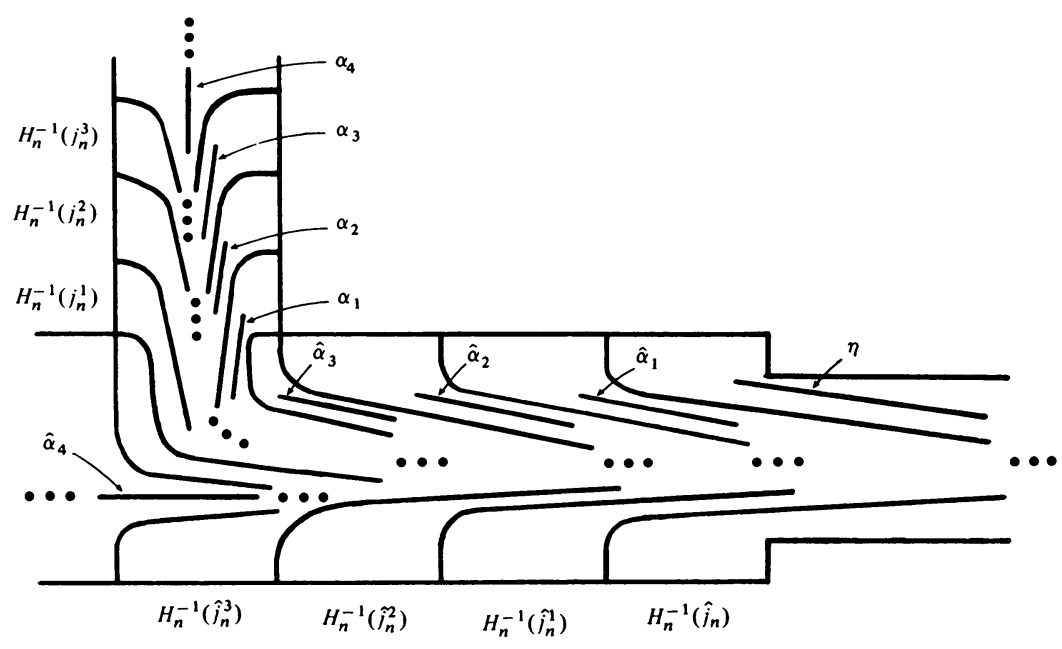

FIGURE 3 
An important observation is that the alteration done with respect to the $\eta$ 's guarantees that $(6.1 .5)^{n}$ holds for pairs $j_{n}$ and $j_{n-1}$ with $j_{n} \cap j_{n-1} \neq \varnothing$; in particular, the above containment holds if $j_{n} \cap j_{n-1} \neq \varnothing$.

Finally, the reader is urged to first read the following proof assuming that $Y$ is a 2-manifold; once one understands the proof for this case it is an easy matter to understand the general case.

Proof of Proposition (6.1). Let $\left\{\mathcal{G}_{i}\right\}_{i=1}^{\infty}$ be a sequence of $f$-admissible closed brick partitions of $Y$ with $\mathcal{G}_{i}$ refining $\mathcal{G}_{i-1}$ and with $\lim _{i \rightarrow \infty} \mu\left(\mathcal{G}_{i}\right)=0$. Let $c_{1}$ be such that $\mu\left(\mathcal{G}_{c_{1}}\right)<\varepsilon / 3$; let $J_{1}=\mathcal{G}_{c_{1}}$ and let $H_{1}=f$. Let us assume that the metric on $M$ is such that $(6.1 .4)^{1}$ holds; this completes the $n=1$ stage.

In (6.2)-(6.7), we give in detail the construction of the $n=2$ stage; in (6.8) we indicate that the inductive step, going from the $n$th stage to the $(n+1)$ st stage, can be done by modifying the construction of the $n=2$ stage. In (6.3)s (the subscript $s$ will be explained later) we carefully construct several collections of arcs and in (6.5) and (6.6) we modify $H_{1}$ by "pulling" various sets over these arcs in order to obtain $\mathrm{H}_{2}$ (recall the proof of Proposition (4.2)).

(6.2) Let $\delta=1 / 2^{2}$ and let $a_{2}>c_{1}$ be such that:

(6.2.1) $\mu\left(\mathcal{G}_{a_{2}}\right)<\xi_{2} / 2^{2}$ and for each $g \in \mathcal{G}_{a_{2}}, \cap\left\{j_{1} \in J_{1} \mid j_{1}\right.$ meets $\left.\operatorname{st}^{2}\left(g, \mathcal{G}_{a_{2}}\right)^{*}\right\} \neq \varnothing$. The partition $\mathcal{G}_{a_{2}}$ is used to control the construction so that condition $(6.1 .3)^{2}$ will hold.

Let $\mathcal{Q}, \mathfrak{T}_{1}, \ldots, \mathfrak{K}_{I(\mathscr{Q})}$ be the filtration obtained from the brick partition $J_{1}=\left\{j_{1}^{1}, \ldots, j_{1}^{T}\right\}$ (see $\$ 2$ ). We are now going to build $J_{2}$ inductively, $s=1, \ldots, I(\mathbb{Q})$, using the $\Re_{s}$ 's; at the same time we will be constructing collections of arcs which will be used later to modify $H_{1}$ in order to obtain $H_{2}$ (we emphasize that no modifying of $H_{1}$ is done until (6.5)). The subscript $s$ in (6.3) refers to the $s$ th stage in the induction, $s=1, \ldots, I(\mathbb{Q})$.

(6.3) $)_{1}$ The $s=1$ stage: Let $3 \tau_{1}=\min \left\{d\left(|x|, Y-j_{1}^{u_{1}} \cup \cdots \cup j_{1}^{u_{k}}\right) \mid x=\right.$ $\left.\left(u_{1}, \ldots, u_{k}\right) \in \mathfrak{T}_{1}\right\}$; recall that if $x, y \in \mathfrak{T}_{1}$ and $x \neq y$, then $|x| \cap|y|=\varnothing$. Let $c_{2}^{1}>a_{2}$ be such that:

(6.3.1) $\mu\left(\mathcal{G}_{1}\right)<\tau_{1}$ and for each $x \in \mathscr{T}_{1}$, letting $J_{2}^{x}=\operatorname{st}\left(|x|, \mathcal{G}_{c_{2}^{1}}\right),\left(J_{2}^{x}\right)^{*} \subseteq$ $\operatorname{int}\left(\operatorname{st}\left(|x|, \mathcal{G}_{a_{2}}\right)^{*}\right)$.

Let $J_{2}^{1}=\left\{J_{2}^{x} \mid x \in \Re_{1}\right\}^{*} ; J_{2}^{1}$ will be a subset of $J_{2}$.

Let $\Gamma_{1}=\left\{\left(j_{2}, u\right) \mid\right.$ for some $x \in \mathfrak{T}_{1}, j_{2} \in J_{2}^{x}$ and $u$ appears in $x$; i.e., $\left.j_{2} \cap j_{2}^{u} \neq \varnothing\right\}$. For each $\gamma=\left(j_{2}, u\right) \in \Gamma_{1}$, let $Q_{\gamma} \subseteq H_{1}^{-1}\left(\operatorname{int}\left(j_{2}\right)\right)$ be an open $m$-ball with $\operatorname{diam}\left(Q_{\gamma}\right)<\delta / 4$ and let

$$
\eta_{\gamma}=\eta_{\gamma}^{1} \cup \cdots \cup \eta_{\gamma}^{b_{\gamma}} \subseteq H_{1}^{-1}\left(\operatorname{int}\left(j_{2} \cup j_{1}^{u}\right)\right)
$$

be an arc satisfying:

$$
\begin{aligned}
& (6.3 .4)_{1} \eta_{\gamma}^{1} \subseteq Q_{\gamma} ; \eta_{\gamma}(1) \in H_{1}^{-1}\left(\operatorname{int}\left(j_{1}^{u}\right)\right) \subseteq N_{\delta / 2}\left(\eta_{\gamma}\right) ; \text { for } i=1, \ldots, b_{\gamma}, \\
& \operatorname{diam}\left(\eta_{\gamma}^{i}\right)<\delta / 4
\end{aligned}
$$


In addition, we assume that the $\eta_{\gamma} \cup \bar{Q}_{\gamma}$ 's are pairwise disjoint. Let $E_{1}=$ $\max \left\{b_{\gamma} \mid \gamma \in \Gamma_{1}\right\}$ and let $\mathcal{E}_{1}=E_{1}+1$; for each $j_{2} \in J_{2}^{1}$, let

$$
\mathscr{P}_{j_{2}}=\left\{Q_{\gamma} \mid \gamma \in \Gamma_{1} \text { and } Q_{\gamma} \subseteq H_{1}^{-1}\left(\operatorname{int}\left(j_{2}\right)\right) \text {; i.e., } \gamma=\left(j_{2}, u\right)\right\} \text {. }
$$

(6.3) The inductive step, $s=2, \ldots, I(\mathbb{Q})$ : For each $x=\left(u_{1}, \ldots, u_{k}\right) \in$ $\mathfrak{N}_{s}$, let

$$
|\hat{x}|=\operatorname{cl}\left(|x|-\left(J_{2}^{1} \cup \cdots \cup J_{2}^{s-1}\right)^{*}\right)
$$

and let

$$
\begin{aligned}
D^{x}= & \left(Y-j_{1}^{u_{1}} \cup \cdots \cup j_{1}^{u_{k}}\right) \\
& \cup\left\{\left(J_{i}^{y}\right)^{*} \mid y \in \Re_{1} \cup \cdots \cup \Re_{s-1} \text { and } y \nless x\right\}^{*} .
\end{aligned}
$$

Let $3 \tau_{s}=\min \left\{d\left(|\hat{x}|, D^{x}\right) \mid x \in \Re_{s}\right\}$ and let $c_{2}^{s}>c_{2}^{s-1}$ be such that:

(6.3.1) $)_{s} \mu\left(\mathcal{G}_{c_{2}^{s}}\right)<\tau_{s}$ and, for $x \in \mathscr{T}_{s}$, letting

$$
J_{2}^{x}=\mathbf{s t}\left(|\hat{x}|, \mathcal{G}_{c_{2}^{s}}\right)-\mathrm{st}^{0}\left(\left(J_{2}^{1} \cup \cdots \cup J_{2}^{s-1}\right)^{*}, \mathcal{G}_{c_{2}^{s}}\right),
$$

$\left(J_{2}^{x}\right)^{*} \subseteq \operatorname{int}\left(\operatorname{st}\left(|\hat{x}|, \mathcal{G}_{a_{2}}\right)^{*}\right)$.

(6.3.2)s For each $y \in \Re_{1} \cup \cdots \cup \Re_{s-1}$,

$$
\mathrm{st}^{\mathbb{E}_{s-1}}\left(\left(J_{2}^{y}\right)^{*}, \mathcal{G}_{c_{2}^{s}}\right)^{*} \subseteq \operatorname{int}\left(\operatorname{st}\left(|\hat{y}|, \mathcal{G}_{a_{2}}\right)^{*}\right) \text {. }
$$

(6.3.3) setting $J_{2}^{s}=\left\{J_{2}^{x} \mid x \in \Re_{s}\right\}^{*}$, for each $j_{2} \in J_{2}^{s}, \quad \cap\left\{j_{2}^{\prime} \in J_{2}^{1}\right.$ $\cup \cdots \cup J_{2}^{s-1} \mid j_{2}^{\prime}$ meets st $\left.{ }^{\mathcal{E}_{s-1}}\left(j_{2}, \mathcal{G}_{c_{2}^{s}}\right)^{*}\right\} \neq \varnothing$.

Let $\Gamma_{s}=\left\{\left(j_{2}, u\right) \mid\right.$ for some $x \in \mathfrak{R}_{s}, j_{2} \in J_{2}^{x}$ and $u$ appears in $x$; i.e., $\left.j_{2} \cap j_{1}^{u} \neq \varnothing\right\}$. For each $\gamma=\left(j_{2}, u\right) \in \Gamma_{s}$, let $Q_{\gamma} \subseteq H_{1}^{-1}\left(\operatorname{int}\left(j_{2}\right)\right)$ be an open $m$ ball with $\operatorname{diam}\left(Q_{\gamma}\right)<\delta / 4$ and let

$$
\eta_{\gamma}=\eta_{\gamma}^{1} \cup \cdots \cup \eta_{\gamma}^{b_{\gamma}} \subseteq H_{1}^{-1}\left(\operatorname{int}\left(j_{2} \cup j_{1}^{u}\right)\right)
$$

be an arc satisfying:

(6.3.4) $)_{s} \eta_{\gamma}^{1} \subseteq Q_{\gamma} ; \quad \eta_{\gamma}(1) \in H_{1}^{-1}\left(\operatorname{int}\left(j_{1}^{u}\right)\right) \subseteq N_{\delta / 2}\left(\eta_{\gamma}\right) ;$ for $i=1, \ldots, b_{\gamma}$, $\operatorname{diam}\left(\eta_{\gamma}^{i}\right)<\delta / 4$.

For each $j_{2} \in J_{2}^{1} \cup \cdots \cup J_{2}^{s-1}$, define

$$
\Omega_{j_{2}}^{s}=\Omega_{j_{2}}^{s, 1} \cup \Omega_{j_{2}}^{s, 2} \cup \cdots \cup \Omega_{j_{2}}^{s, \mathscr{E}_{s-1}}
$$

as follows:

$$
\begin{aligned}
\Omega_{j_{2}}^{s, 1} & =\left\{\text { chains }\left(j_{2}^{1}\right) \mid j_{2}^{1} \in J_{2}^{s} \text { and } j_{2} \cap j_{2}^{1} \neq \varnothing\right\} ; \\
\Omega_{j_{2}}^{s, 2} & =\left\{\operatorname{chains}\left(j_{2}^{1}, j_{2}^{2}\right) \mid j_{2}^{1}, j_{2}^{2} \in J_{2}^{s} \text { and } j_{2} \cap j_{2}^{1} \neq \varnothing\right\} ; \ldots ; \\
\Omega_{j_{2}}^{s \mathcal{E}_{s-1}} & =\left\{\operatorname{chains}\left(j_{2}^{1}, j_{2}^{2}, \ldots, j_{2}^{\mathbb{E}_{s-1}}\right) \mid j_{2}^{1}, j_{2}^{2}, \ldots, j_{2}^{\mathcal{E}_{s-1}} \in J_{2}^{s} \text { and } j_{2} \cap j_{2}^{1} \neq \varnothing\right\} .
\end{aligned}
$$

Let $j_{2} \in J_{2}^{1} \cup \cdots \cup J_{2}^{s-1}$. For each $\omega_{1}=\left(j_{2}^{1}\right) \in \Omega_{j_{2}}^{s, 1}$ and for each $p \in$ $\mathscr{P}_{j_{2}}$, let

$$
\Sigma_{\omega_{1, p}}^{s}=\left(Q_{\omega_{1}, p}, \alpha_{\omega_{1, p}}=\alpha_{\omega_{1}, p}^{1} \cup \cdots \cup \alpha_{\omega, p}^{d_{\omega, p}}\right)
$$


be such that:

$(6.3 .5)_{s}^{\omega_{1}, p} Q_{\omega_{1}, p} \subseteq H_{1}^{-1}\left(\operatorname{int}\left(j_{2}^{1}\right)\right)$ is an open $m$ ball with $\operatorname{diam}\left(Q_{\omega_{1}, p}\right)<\delta / 4$; $\alpha_{\omega_{1}, p} \subseteq H_{1}^{-1}\left(\operatorname{int}\left(j_{2} \cup j_{2}^{1}\right)\right)$ is an arc with $\alpha_{\omega_{1}, p}^{1} \subseteq Q_{\omega_{1}, p}$, with $\alpha_{\omega_{1}, p}(1) \in p$, and with $\operatorname{diam}\left(\alpha_{\omega_{1, p}}^{k}\right)<\delta / 4$ for $1 \leqslant k \leqslant d_{\omega_{1, p}}$.

Inductively, $i=2, \ldots, \varepsilon_{s-1}$, for each $\omega_{i}=\left(j_{2}^{1}, \ldots, j_{2}^{i}\right) \in \Omega_{j_{2}}^{s, i}$ and for each $p \in \mathscr{P}_{j_{2}}$, let

$$
\Sigma_{\omega_{i} p}^{s}=\left(Q_{\omega_{i} p}, \alpha_{\omega_{i} p}=\alpha_{\omega_{i} p}^{1} \cup \cdots \cup \alpha_{\omega_{i} p}^{d_{\omega_{j} p}}\right)
$$

be such that:

(6.3.5) $)_{s}^{\omega_{i} p} Q_{\omega_{i}, p} \subseteq H_{1}^{-1}\left(\operatorname{int}\left(j_{2}^{i}\right)\right)$ is an open $m$ ball with $\operatorname{diam}\left(Q_{\omega_{i}, p}\right)<\delta / 4$; $\alpha_{\omega_{i}, p} \subseteq H_{1}^{-1}\left(\operatorname{int}\left(j_{2}^{i-1} \cup j_{2}^{i}\right)\right)$ is an arc with $\alpha_{\omega_{i} p}^{1} \subseteq Q_{\omega_{i}, p}$, with $\alpha_{\omega_{i}, p}(1) \in Q_{\omega_{i-1}, p}$ where $\omega_{i-1}=\left(j_{2}^{1}, \ldots, j_{2}^{i-1}\right)$, and with $\operatorname{diam}\left(\alpha_{\omega_{1}, p}^{k}\right)<\delta / 4$ for $k=$ $1, \ldots, d_{\omega_{i}, p}$.

Let $E_{s}=\max \left\{\left\{b_{\gamma} \mid \gamma \in \Gamma_{s}\right\} \cup\left\{d_{\omega, p} \mid j_{2} \in J_{2}^{1} \cup \cdots \cup J_{2}^{s-1}, \omega \in \Omega_{j_{2}}^{s}\right.\right.$, and $\left.\left.p \in \mathscr{P}_{j_{2}}\right\}\right\}$ and let $\mathcal{E}_{s}=1+\mathcal{E}_{1}+\mathcal{E}_{1} \cdot E_{2}+\cdots+\mathcal{E}_{s-1} \cdot E_{s}$. For each $j_{2} \in$ $J_{2}^{s}$, let $\mathscr{P}_{j_{2}}=\left\{Q_{\gamma} \mid \gamma \in \Gamma_{s}\right.$ and $Q_{\gamma} \subseteq H_{1}^{-1}\left(\operatorname{int}\left(j_{2}\right)\right)$; i.e., $\left.\gamma=\left(j_{2}, u\right)\right\} \cup\left\{Q_{\omega, p} \mid \omega\right.$ $=\left(j_{2}^{1}, \ldots, j_{2}^{r}\right) \in \Omega_{j_{2}^{\prime}}^{s}, p \in \mathscr{P}_{j_{2}^{\prime}}$, and $j_{2}=j_{2}^{r}$; i.e., $\left.Q_{\omega, p} \subseteq H_{1}^{-1}\left(\operatorname{int}\left(j_{2}\right)\right)\right\}$. This completes the induction on $s$.

(6.4) Let $J_{2}=J_{2}^{1} \cup \cdots \cup J_{2}^{I(\Theta)}$. A final condition on the choices of arcs and $m$-balls in (6.3) $-(6.3)_{I(\mathscr{C})}$ is that there be no unnecessary intersections. More specifically: (i) all arcs constructed are to be pairwise disjoint; (ii) all the $m$-balls specified are to have pairwise disjoint closures; (iii) the only intersections between arcs and $m$ balls are those necessitated by (6.3.4) $s$ and (6.3.5) for $s=1, \ldots, I(\mathbb{Q})$.

(6.5) The mapping $H_{2}$ will be obtained from $H_{1}$ by a finite number of alterations; we will now give a detailed description of one of these alterations.

Let $j_{1}^{u} \in J_{1}$ and let $j_{2} \in \operatorname{st}\left(j_{1}^{u}, J_{2}\right)$; let $v_{0}$ be such that $j_{2} \in J_{2}^{v_{0}}$. Note that $\gamma=\left(j_{2}, u\right) \in \Gamma_{v_{0}}$. For $l=1, \ldots, t$ let $v_{l}$ be an integer and let $\left(j_{2}^{1_{1}}, \ldots, j_{2}^{r_{1}}\right)$ be a chain such that:

(6.5.1) $1 \leqslant v_{0} \lesseqgtr v_{1} \lesseqgtr \cdots \lesseqgtr v_{t} \leqslant I(\mathbb{Q}) ;\left(j_{2}^{1_{1}}, \ldots, j_{2}^{r_{1}}\right) \in \Omega_{j_{2}}^{v_{1}} ;$ and, for $l=$ $2, \ldots, t$,

$$
\left(j_{2}^{1_{1}}, \ldots, j_{2}^{r_{t}}\right) \in \Omega_{j_{2}^{\prime \prime-1}}^{v_{t}} \text {. }
$$

In particular, $\left(j_{2}, j_{2}^{1_{1}}, \ldots, j_{2}^{r_{1}}, \ldots, j_{2}^{1_{t}}, \ldots, j_{2}^{r_{t}}\right)$ is a chain.

For $l=1, \ldots, t$ and $i=1, \ldots, r_{l}$, let $\omega_{i}^{l}=\left(j_{2}{ }^{1}, \ldots, j_{2}^{i}\right)$; from the data in (6.5.1) we obtain the following. $Q_{\gamma}$,

(6.5.2) $R_{0}=\left\{\left(Q_{\gamma}, \eta_{\gamma}=\eta_{\gamma}^{1} \cup \cdots \cup \eta_{\gamma}^{b_{\gamma}}\right)\right\}$ where $\gamma=\left(j_{2}, u\right)$; letting $p_{1}=$

$$
\begin{aligned}
& R_{1}=\left\{\Sigma_{\omega_{1}, p_{1}}^{v_{1}}=\left(Q_{\omega_{1}^{1}, p_{1}}, \alpha_{\omega_{1}^{1}, p_{1}}=\alpha_{\omega_{1}^{1} p_{1}}^{1} \cup \cdots \cup \alpha_{\omega_{1}^{1} p_{1}}^{d_{\omega_{1} p_{1}}}\right), \ldots,\right. \\
& \left.\Sigma_{\omega_{r}, p_{1}}^{v_{1}}=\left(Q_{\omega_{r, 1}^{1}, p_{1}}, \alpha_{\omega_{r, 1}^{1}, p_{1}}=\alpha_{\omega_{r}^{1}, p_{1}}^{1} \cup \cdots \cup \alpha_{\substack{\omega_{r}, p_{1} \\
\omega_{r}^{1}, p_{1}}}^{d_{1}}\right)\right\} ;
\end{aligned}
$$


for $l=2, \ldots, t$, letting $p_{l}=Q_{\omega_{r_{l-1}}^{l-1} p_{l-1}}$,

$$
\begin{aligned}
& R_{l}=\left\{\Sigma_{\omega_{1}, p_{l}}^{v_{l}}=\left(Q_{\omega_{1}^{l}, p_{t-1}}, \alpha_{\omega_{1}^{l}, p_{l}}=\alpha_{\omega_{1}^{\prime}, p_{l}}^{1} \cup \cdots \cup \alpha_{\omega_{1}^{\prime}, p_{l}}^{d_{\omega^{\prime}, p_{l}}}\right), \ldots,\right.
\end{aligned}
$$

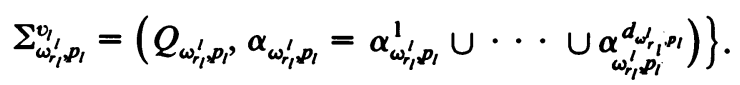

$N\left(\eta_{\gamma}\right)$ and $N\left(\alpha_{\omega, p}\right)$ will denote "small" neighborhoods of the arcs $\eta_{\gamma}$ and $\alpha_{\omega, p}$, respectively. Since all the arcs involved are pairwise disjoint, we can assume that all such neighborhoods are pairwise disjoint; in addition, if $\gamma=\left(j_{2}, u\right)$; then $N\left(\eta_{\gamma}\right) \subseteq H_{1}^{-1}\left(\operatorname{int}\left(j_{2} \cup j_{1}^{u}\right)\right)$ and if $\omega=\left(j_{2}^{1}, \ldots, j_{2}^{r}\right) \in \Omega_{j_{2}}^{s}$, then $N\left(\alpha_{\omega, p}\right) \subseteq H_{1}^{-1}\left(\operatorname{int}\left(j_{2} \cup j_{2}^{1}\right)\right)$ when $r=1$ and $N\left(\alpha_{\omega, p}\right) \subseteq H_{1}^{-1}\left(\operatorname{int}\left(j_{2}^{r-1}\right.\right.$ $\left.\cup j_{2}^{r}\right)$ ) when $r \gtrless 1$. Furthermore, at various points during the altering of $H_{1}$ and the verifying that $J_{2}$ and $H_{2}$ satisfy $(6.1 .1)^{2}-(6.1 .5)^{2}$ we will further restrict the size of these neighborhoods.

Before describing the alteration, it is convenient to simplify the notation describing the data in (6.5.2). Let

$$
\begin{aligned}
\left(\beta_{0}, \beta_{1}, \ldots, \beta_{D}\right) & \\
& =\left(\eta_{\gamma}, \alpha_{\omega_{1}, p_{1}}, \ldots, \alpha_{\omega_{r}, p_{1}}, \alpha_{\omega_{1}^{2}, p_{2}}, \ldots, \alpha_{\omega_{r_{2}}, p_{2}}, \ldots, \alpha_{\omega_{1}^{1}, p_{1}}, \ldots, \alpha_{\omega_{r_{1}}^{\prime} p_{1}}\right) ;
\end{aligned}
$$

let $Q_{\beta_{0}}=Q_{\gamma}$, and for $\beta_{i^{\prime}}=\alpha_{\omega_{i}^{\prime}, p_{1}}$, let $Q_{\beta_{i}^{\prime}}=Q_{\omega_{i}^{\prime}, p_{i}}$. The arc $\beta_{0}$ is the union of $\eta_{\gamma}^{\text {'s }}$ and each of $\beta_{1}, \beta_{2}, \ldots, \beta_{D}$ is the union of $\alpha_{\omega p}^{i}$ 's; let $\lambda_{1}, \lambda_{2}, \ldots, \lambda_{B}$ be the collection of these subarcs of the $\beta_{i}$ 's ordered as follows:

$$
\begin{aligned}
\beta_{D} & =\lambda_{1} \cup \lambda_{2} \cup \cdots \cup \lambda_{q_{D}}, \\
\beta_{D-1} & =\lambda_{q_{D}+1} \cup \lambda_{q_{D}+2} \cup \cdots \cup \lambda_{q_{D-1}}, \ldots, \\
\beta_{1} & =\lambda_{q_{2}+1} \cup \cdots \cup \lambda_{q_{1}}, \\
\beta_{0} & =\lambda_{q_{1}+1} \cup \cdots \cup \lambda_{B} .
\end{aligned}
$$

Using the above data alter $H_{1}$ as follows. Let $\left\{h_{t}\right\}_{t \in[0,1]}$ be an isotopy of $M$ with $h_{0}=$ identity and with $\operatorname{supp}\left(\left\{h_{t}\right\}_{t \in[0,1]} \subseteq N\left(\beta_{0}\right)\right.$ such that $\left\{h_{t}^{-1}\right\}_{t \in[0,1]}$ "pulls" a small neighborhood of $\beta_{0}(0)$ contained in $Q_{\beta_{0}}$ over the entire arc $\beta_{0}$. Successively, $i=1, \ldots, D$, let $\left\{h_{t}\right\}_{t \in[2 i, 2 i+1]}$ be an isotopy of $M$ with $h_{2 i}=$ identity and with

$$
\operatorname{supp}\left(\left\{h_{t}\right\}_{t \in[2 i, 2 i+1]}\right) \subseteq N\left(\beta_{i}\right) \cup\left(\bigcup_{q=0}^{i-1}\left(Q_{\beta_{q}} \cup N\left(\beta_{q}\right)\right)\right)
$$

such that $\left\{h_{t}^{-1}\right\}_{t \in[2 i, 2 i+1]}$ "pulls" a small neighborhood of $\beta_{i}(0)$ contained in $Q_{\beta_{i}}$ over the entire arc $\beta_{i}$ and then "pulls" the neighborhood "alongside" $\lambda_{\beta_{i}} \cup \lambda_{\beta_{i+1}} \cup \cdots \cup \lambda_{B-i}$. Let $\hat{H}_{1}=H_{1} \circ h_{2 D+1} \circ h_{2(D-1)+1} \circ \cdots \circ h_{1}$.

Essentially, $H_{2}$ is obtained from $H_{1}$ by making the above alteration for all possible choices of data satisfying (6.5.1) and (6.5.2); however, there is the minor problem that different sets of data may well "overlap". More precisely, 
two sets of data $\left(\beta_{0}, \ldots, \beta_{D}\right)$ and $\left(\beta_{0}^{\prime}, \ldots, \beta_{D^{\prime}}^{\prime}\right)$ are said to overlap provided that there are $q$ and $q^{\prime}$ with $\beta_{q}=\beta_{q^{\prime}}^{\prime}$; in this case, it follows that $\beta_{q+1}=\beta_{q^{\prime}+1}^{\prime}$, $\beta_{q+2}=\beta_{q^{\prime}+2}^{\prime}, \ldots, \beta_{D}=\beta_{D^{\prime}}^{\prime}$. (This last statement can be verified using the following two facts. First, if $\beta_{q}=\beta_{q^{\prime}}^{\prime}$, then either $q=q^{\prime}=0$ and $\beta_{q}=\beta_{q^{\prime}}^{\prime}=$ $\eta_{\gamma}$ or

$$
\beta_{q}=\alpha_{\omega_{i}^{k}, p_{k}}, \quad \beta_{q^{\prime}}^{\prime}=\alpha_{\hat{\omega}_{i^{\prime}}^{k^{\prime}, k^{\prime}}}
$$

and, therefore, $\omega_{i}^{k}=\hat{\omega}_{i^{\prime \prime}}^{k^{\prime}}$ and $p_{k}=p_{k^{\prime}}^{\prime}$. Second, $\omega_{i}^{k}$ (resp., $\hat{\omega}_{i^{\prime}}^{k^{\prime}}$ ) and the conditions in (6.5.2) that $p_{1}=Q_{\gamma}$ (resp., $p_{1}^{\prime}=Q_{\gamma^{\prime}}^{\prime}$ ) and, for $l=2, \ldots, t$, $p_{l}=Q_{\omega_{r_{-1}}^{\prime-1} p_{l-1}}$ (resp., $l=2, \ldots, t^{\prime}, p_{l}^{\prime}=Q_{\omega_{l_{-1}}^{\prime l}, p_{l-1}^{\prime}}^{\prime}$ ) completely determine $\beta_{q+1}, \ldots, \beta_{D}$ (resp., $\left.\beta_{q^{\prime}+1}^{\prime}, \ldots, \beta_{D^{\prime}}^{\prime}\right)$.)

The alterations described above have two important features. First each set "pulled alongside" various arcs meets only that arc which it is "pulled over". Second, the sets "pulled alongside" various arcs are "pulled straight alongside" the arcs; i.e., the sets are not permitted to "wiggle back and forth" along the arcs. (The second feature and the facts that the $\operatorname{diam}\left(Q_{\beta_{i}}\right)$ 's are less than $\delta / 4$ and that the $\operatorname{diam}\left(\lambda_{i}\right)$ 's are less than $\delta / 4$ imply that each successive set "pulled" alongside the $\lambda_{i}$ 's is within $\delta / 2$ of the previous one. At least this will be the case if the $N\left(\beta_{i}\right)$ 's are chosen small enough; we assume that they have been so chosen.)

One way of obtaining $\mathrm{H}_{2}$ is to make the indicated alteration for all possible choices of data in (6.5.1) and (6.5.2). The reader should observe that conditions $(6.1 .2)^{2}$ and $(6.1 .5)^{2}$ can be verified by studying the role of the $\eta_{\gamma}$ 's. Condition $(6.1 .3)^{2}$ is relatively easy to verify but it is convenient to delay doing so until later. We are left with condition (6.1.4) ${ }^{2}$; certainly, achieving $(6.1 .4)^{2}$ is the central difficulty faced throughout the construction. At this point, we suggest that the reader compare condition $(6.1 .4)^{2}$ and the alterations outlined in this section in order to get a "sense" that (6.1.4) ${ }^{2}$ holds.

Thus far we have attempted to give a global description $\mathrm{H}_{2}$; in the next section, we will obtain $H_{2}$ from $H_{1}$ in a more "controlled" manner thereby facilitating the verification of condition (6.1.4) ${ }^{2}$. The method of the next section will also illuminate the role of the filtration $\mathfrak{N}_{1}, \ldots, \mathfrak{N}_{I(\mathscr{Q})}$.

(6.6) We are now going to alter $H_{1}$ inductively for $s=1, \ldots, I(Q)$. Recall that the $N\left(\eta_{\gamma}\right)$ 's and the $N\left(\alpha_{\omega_{i}, p}\right)$ 's are small neighborhoods of the $\eta_{\gamma}$ 's and $\alpha_{\omega_{i}, p}$ 's, respectively.

$s=1$ : For each $\gamma=\left(j_{2}, u\right) \in \Gamma_{1}$, alter $H_{1}$ by using an isotopy of $M$ with support contained in $Q_{\gamma} \cup N\left(\eta_{\gamma}\right)$ to "pull" a small neighborhood of $\eta_{\gamma}(0)$ contained in $Q_{\gamma}$ over the entire arc $\eta_{\gamma}$. Let $H_{1,1}$ denote the mapping so obtained. It is easily verified that $H_{1,1}$ satisfies $(6.1 .2)^{2}$ and $(6.1 .5)^{2}$ for $j_{2} \in J_{2}^{1}$. We now check that $H_{1,1}$ satisfies (6.1.4) ${ }^{2}$ for pairs $j_{2}, j_{2}^{\prime} \in J_{2}^{1}$ with $j_{2} \cap j_{2}^{\prime} \neq \varnothing$. Let $x=\left(u_{1}, \ldots, u_{k}\right) \in \Re_{1}$ with $j_{2}, j_{2}^{\prime} \in J_{2}^{x}$; then we have that 


$$
H_{1,1}^{-1}\left(\operatorname{int}\left(j_{2}\right)\right) \subseteq H_{1}^{-1}\left(\operatorname{int}\left(j_{1}^{u_{1}} \cup \cdots \cup j_{1}^{u_{k}}\right)\right) \subseteq N_{\delta}\left(H_{1,1}^{-1}\left(\operatorname{int}\left(j_{2}^{\prime}\right)\right)\right) .
$$

(The latter containment follows from $(6.1 .5)^{2}$ and the fact that $J_{1}$ is $H_{1}$ admissible.)

$s=2$ : For each $\gamma=\left(j_{2}, u\right) \in \Gamma_{2}$, alter $H_{1,1}$ by using an isotopy of $M$ with support contained in $Q_{\gamma} \cup N\left(\eta_{\gamma}\right)$ to "pull" a small neighborhood of $\eta_{\gamma}(0)$ contained in $Q_{\gamma}$ over the entire arc $\eta_{\gamma}$. Let $H_{1,2}^{\prime}$ denote the mapping so obtained. It is easily verified that $H_{1,2}^{\prime}$ satisfies $(6.1 .2)^{2}$ and $(6.1 .5)^{2}$ for $j_{2} \in J_{2}^{1} \cup J_{2}^{2}$; however, $H_{1,2}^{\prime}$ does not satisfy $(6.1 .4)^{2}$ for all pairs $j_{2}, j_{2}^{\prime} \in J_{2}^{1} \cup$ $J_{2}^{2}$ with $j_{2} \cap j_{2}^{\prime} \neq \varnothing\left(H_{1,2}^{\prime}\right.$ does satisfy $(6.1 .4)^{2}$ for such pairs $\left.j_{2}, j_{2}^{\prime} \in J_{2}^{1}\right)$.

Let $\omega=\left(j_{2}^{1}, \ldots, j_{2}^{r}\right) \in \Omega_{j_{2}}^{2}$ for some $j_{2} \in J_{2}^{1}$ and let $p \in \mathscr{P}_{j_{2}}$; necessarily, $p=Q_{\gamma}$ for some $\gamma=\left(j_{2}, u\right) \in \Gamma_{1}$. Obtain a set of data as in (6.5.1) by letting $v_{0}=1, v_{1}=2(l=1)$, and $\left(j_{2}^{1_{1}}, \ldots, j_{2}^{r_{1}}\right)=\omega$. Extract the data of (6.5.2) from the above data and alter $H_{1,2}^{\prime}$ as outlined in (6.5); observe that part of the alteration was done while altering $H_{1}$ to get $H_{1,1}$. The mapping $H_{1,2}$ is obtained by making the above alteration for all triples $j_{2} \in J_{2}^{1}, \omega \in \Omega_{j_{2}}^{2}$, and $p \in \mathscr{P}_{j_{2}}$ (if the present data $j_{2}, \omega, p$ overlaps with previous data $j_{2}^{\prime}, \omega^{\prime}, p$, then part or all of the alteration based on $j_{2}, \omega, p$ will have been done; in that case, complete the remainder of the alteration).

Certainly, $H_{1,2}$ satisfies (6.1.2) $)^{2}$ and $(6.1 .5)^{2}$ for $j_{2} \in J_{2}^{1} \cup J_{2}^{2}$ and there is no difficulty in doing the above altering of $H_{1,2}^{\prime}$ so that $H_{1,2}$ satisfies (6.1.4) ${ }^{2}$ for $j_{2}, j_{2}^{\prime} \in J_{2}^{1}$. That $(6.1 .4)^{2}$ holds for pairs $j_{2}, j_{2}^{\prime} \in J_{2}^{1} \cup J_{2}^{2}$ can be checked as follows.

Case 1. $j_{2} \in J_{2}^{2}$ and $j_{2}^{\prime} \in J_{2}^{1}$ with $j_{2} \cap j_{2}^{\prime} \neq \varnothing$.

Let $y \in \Re_{1}$ with $j_{2}^{\prime} \in J_{2}^{y}$ and let $x \in \mathscr{T}_{2}$ with $j_{2} \in J_{2}^{x}$. Let $x=$ $\left(u_{1}, \ldots, u_{k}\right)$ and $y=\left(u_{1}^{\prime}, \ldots, u_{k^{\prime}}^{\prime}\right)$; it follows from $(6.3 .1)_{2}$ that $x \geqslant y$ and, therefore, that $\left\{u_{1}, \ldots, u_{k}\right\} \subseteq\left\{u_{1}^{\prime}, \ldots, u_{k^{\prime}}^{\prime}\right\}$.

We first show that

$$
H_{1,2}^{-1}\left(\operatorname{int}\left(j_{2}\right)\right) \subseteq N_{\delta}\left(H_{1,2}^{-1}\left(\operatorname{int}\left(j_{2}^{\prime}\right)\right)\right) ;
$$

since $H_{1}^{-1}\left(\operatorname{int}\left(j_{1}^{u_{1}^{\prime}} \cup \cdots \cup j_{1}^{u_{k^{\prime}}^{\prime}}\right)\right) \subseteq N_{\delta / 2}\left(H_{1,2}^{-1}\left(\operatorname{int}\left(j_{2}^{\prime}\right)\right)\right)$, it suffices to show that

$$
H_{1,2}^{-1}\left(\operatorname{int}\left(j_{2}\right)\right) \subseteq H_{1}^{-1}\left(\operatorname{int}\left(j_{1}^{u_{1}^{\prime}} \cup \cdots \cup j_{1}^{u_{k^{\prime}}^{\prime}}\right)\right)
$$

Since a chain in any $\Omega_{j_{2}^{\prime \prime}}^{2}$ has length at most $\varepsilon_{1}$, it follows from (6.3.3) $)_{2}$ that if $j_{2}$ appears in a chain $\omega \in \Omega_{j_{2}^{\prime \prime}}^{2}$ and $\gamma=\left(j_{2}^{\prime \prime}, u^{\prime \prime}\right) \in \Gamma_{1}$, then $u^{\prime \prime} \in\left\{u_{1}^{\prime}, \ldots, u_{k^{\prime}}^{\prime}\right\}$. The containment $H_{1,2}^{-1}\left(\operatorname{int}\left(j_{2}\right)\right) \subseteq H_{1}^{-1}\left(\operatorname{int}\left(j_{1}^{u_{1}^{\prime}} \cup \cdots \cup j_{1}^{u^{\prime}}\right)\right)$ follows by observing the effect of the various alterations on $\mathrm{H}_{1}^{-1}\left(\operatorname{int}\left(j_{2}\right)\right)$.

We now show that

$$
H_{1,2}^{-1}\left(\operatorname{int}\left(j_{2}^{\prime}\right)\right) \subseteq N_{\delta}\left(H_{1,2}^{-1}\left(\operatorname{int}\left(j_{2}\right)\right)\right) .
$$

Since $H_{1,2}^{-1}\left(\operatorname{int}\left(j_{2}^{\prime}\right)\right) \subseteq H_{1}^{-1}\left(\operatorname{int}\left(j_{1}^{u_{1}^{\prime}} \cup \cdots \cup j_{1}^{u_{k^{\prime}}^{\prime}}\right)\right)$, it is enough to show that 


$$
H_{1,2}^{-1}\left(\operatorname{int}\left(j_{2}^{\prime}\right)\right) \cap H_{1}^{-1}\left(\operatorname{int}\left(j_{i}^{u_{i}^{\prime}}\right)\right) \subseteq N_{\delta / 2}\left(H_{1,2}^{-1}\left(\operatorname{int}\left(j_{2}\right)\right)\right) \text { for } i=1, \ldots, k^{\prime}
$$

The latter containment is true for $u_{i}^{\prime} \in\left\{u_{1}, \ldots, u_{k}\right\}$ since for such $u_{i}^{\prime}$ we have that $H_{1}^{-1}\left(\operatorname{int}\left(j_{1}^{u^{\prime}}\right)\right) \subseteq N_{\delta / 2}\left(H_{1,2}^{-1}\left(\operatorname{int}\left(j_{2}\right)\right)\right)$. If $u_{i}^{\prime} \notin\left\{u_{1}, \ldots, u_{k}\right\}$, then $j_{2}^{\prime} \& j_{1}^{u_{i}^{\prime}}$; therefore, $H_{1,2}^{-1}\left(\operatorname{int}\left(j_{2}^{\prime}\right)\right) \cap H_{1}^{-1}\left(\operatorname{int}\left(j_{1}^{u^{\prime}}\right)\right)$ is determined by the fact that $H_{1}$ was altered by pulling $H_{1}^{-1}\left(\operatorname{int}\left(j_{2}^{\prime}\right)\right)$ over $\eta_{\gamma}$ for $\gamma=\left(j_{2}^{\prime}, u_{i}^{\prime}\right)$. Let $\omega=\left(j_{2}\right) \in \Omega_{j_{2}^{\prime}}^{2}$ and let $p=Q_{\gamma}\left(\gamma=\left(j_{2}^{\prime}, u_{i}^{\prime}\right)\right) ; H_{1,2}^{\prime}$ was modified with respect to the data $j_{2}^{\prime}, \omega$, and $p$ and this modification guarantees that $H_{1,2}^{-1}\left(\operatorname{int}\left(j_{2}^{\prime}\right)\right) \cap H_{1}^{-1}\left(\operatorname{int}\left(j_{1}^{u_{1}^{\prime}}\right)\right) \subseteq$ $N_{\delta / 2}\left(H_{1,2}^{-1}\left(\operatorname{int}\left(j_{2}\right)\right)\right)$. (At least the last statement is true if the neighborhood $N\left(\eta_{\gamma}\right)$ is "small enough".)

Case 2. $j_{2}, j_{2}^{\prime} \in J_{2}^{2}$ with $j_{2} \cap j_{2}^{\prime} \neq \varnothing$.

Since the roles of $j_{2}$ and $j_{2}^{\prime}$ are interchangeable, it suffices to show that $H_{1,2}^{-1}\left(\operatorname{int}\left(j_{2}\right)\right) \subseteq N_{\delta}\left(H_{1,2}^{-1}\left(\operatorname{int}\left(j_{2}^{\prime}\right)\right)\right)$. First, observe that $(6.3 .1)_{2}$ implies that there is a unique $y=\left(u_{1}, \ldots, u_{k}\right) \in \mathfrak{T}_{2}$ with $j_{2}, j_{2}^{\prime} \in J_{2}^{y}$. For $i=1, \ldots, k$, we have that

$$
H_{1,2}^{-1}\left(\operatorname{int}\left(j_{2}\right)\right) \cap H_{1}^{-1}\left(\operatorname{int}\left(j_{1}^{u_{i}}\right)\right) \subseteq H_{1}^{-1}\left(\operatorname{int}\left(j_{1}^{u_{i}}\right)\right) \subseteq N_{\delta / 2}\left(H_{1,2}^{-1}\left(\operatorname{int}\left(j_{2}^{\prime}\right)\right)\right) .
$$

We must show that if $u \notin\left\{u_{1}, \ldots, u_{k}\right\}$ and $H_{1,2}^{-1}\left(\operatorname{int}\left(j_{2}\right)\right) \cap H_{1}^{-1}\left(\operatorname{int}\left(j_{1}^{u}\right)\right)$ $\neq \varnothing$, then the intersection is contained in $N_{\delta / 2}\left(H_{1,2}^{-1}\left(\operatorname{int}\left(j_{2}^{\prime}\right)\right)\right)$. This intersection is determined by choices of data $j_{2}^{\prime \prime} \in J_{2}^{1}, \omega=\left(j_{2}^{1}, \ldots, j_{2}^{r}\right) \in \Omega_{j_{2}^{\prime \prime}}^{2}$, and $p \in \mathcal{P}_{j_{2}^{\prime \prime}}$ with $j_{2}^{\prime \prime} \in \operatorname{st}\left(j_{1}^{u}, J_{2}^{1}\right)$ and $j_{2}^{r}=j_{2}$. (Recall from the definition of $\Omega_{j_{2}^{\prime \prime}}^{2}$ that $r \leqslant \varepsilon_{1}$.) If $r=\mathcal{E}_{1}$, then the alteration based on the data $j_{2}^{\prime \prime}, \omega$, and $p$ does not result in $H_{1,2}^{-1}\left(\operatorname{int}\left(j_{2}\right)\right)$ meeting $H_{1}^{-1}\left(\operatorname{int}\left(j_{1}^{u}\right)\right)$; this is true in view of the definition of $\mathcal{E}_{1}$ and in view of the feature in the alteration which has $H_{1,2}^{-1}\left(\operatorname{int}\left(j_{2}^{i}\right)\right)$ being "pulled less and less far" into $H_{1}^{-1}\left(\operatorname{int}\left(j_{1}^{u}\right)\right)$ as $i$ gets larger. If $r<\mathcal{E}_{1}$ and $j_{2}^{\prime} \notin\left\{j_{2}^{1}, \ldots, j_{2}^{r-1}\right\}$, then the data $j_{2}^{\prime \prime}, \hat{\omega}\left(j_{2}^{1}, \ldots, j_{2}^{r}, j_{2}^{\prime}\right) \in \Omega_{j_{2}^{\prime \prime}}^{2}$, and $\stackrel{\neq}{p} \in \mathscr{P}_{j_{2}^{\prime \prime}}$ will result in $H_{1,2}^{-1}\left(\operatorname{int}\left(j_{2}^{\prime}\right)\right)$ being "pulled" to within $\delta / 2$ of that part of $H_{1,2}^{-1}\left(\operatorname{int}\left(j_{2}\right)\right) \cap H_{1}^{-1}\left(\operatorname{int}\left(j_{1}^{u}\right)\right)$ resulting from the alteration done with respect to the data $j_{2}^{\prime \prime}, \omega$, and $p$. If $r<\mathcal{E}_{1}$ and $j_{2}^{\prime}=j_{2}^{t}$ for some $1<t<r-1$, then that part of $H_{1,2}^{-1}\left(\operatorname{int}\left(j_{2}\right)\right)$ "pulfed" into $H_{1}^{-1}\left(\operatorname{int}\left(j_{1}^{u}\right)\right)$ (by the alteration based on the data $j_{2}^{\prime \prime}, \omega$, and $p$ ) was "pulled inside" that part of $H_{1,2}^{-1}\left(\operatorname{int}\left(j_{2}^{\prime}\right)\right)$ "pulled" into $H_{1}^{-1}\left(\operatorname{int}\left(j_{1}^{u}\right)\right.$ ) (by the alteration based on the data $j_{2}^{\prime \prime}$, $\left(j_{2}^{1}, \ldots, j_{2}^{t}\right) \in \Omega_{j_{2}^{\prime \prime}}^{2}$, and $\left.p\right)$. Combining the above two statements, we have that

$$
H_{1,2}^{-1}\left(\operatorname{int}\left(j_{2}\right)\right) \cap H_{1}^{-1}\left(\operatorname{int}\left(j_{1}^{u}\right)\right) \subseteq N_{\delta / 2}\left(H_{1,2}^{-1}\left(\operatorname{int}\left(j_{2}^{\prime}\right)\right)\right)
$$

The inductive step, altering $H_{1, s}$ to get $H_{1, s+1}$ : For each $\gamma \in \Gamma_{s+1}$, alter $H_{1, s}$ with respect to $\eta_{\gamma}$ exactly as $H_{1,1}$ was altered to get $H_{1,2}^{\prime}$; let $H_{1, s+1}^{\prime}$ denote the mapping so obtained. Part of the inductive hypothesis (we have not explicitly displayed the inductive hypothesis) is that $H_{1,5}$ satisfies $(6.1 .2)^{2}$ and (6.1.5) ${ }^{2}$ for $j_{2} \in J_{2}^{1} \cup \cdots \cup J_{2}^{s}$. It is easily verified that $H_{1, s+1}^{\prime}$ satisfies (6.1.2) and $(6.1 .5)^{2}$ for $j_{2} \in J_{2}^{1} \cup \cdots \cup J_{2}^{s+1}$; however, $H_{1, s+1}^{\prime}$ does not satisfy (6.1.4) 
for all pairs $j_{2}, j_{2}^{\prime} \in J_{2}^{1} \cup \cdots \cup J_{2}^{s+1}$ with $j_{2} \cap j_{2}^{\prime} \neq \varnothing$. (Part of the inductive hypothesis is that $H_{1, s}$ satisfies $(6.1 .4)^{2}$ for all pairs $j_{2}, j_{2}^{\prime} \in J_{2}^{1} \cup \ldots \cup J_{2}^{s}$ with $j_{2} \cap j_{2}^{\prime} \neq \varnothing$; it follows easily that $H_{1, s+1}^{\prime}$ satisfies $(6.1 .4)^{2}$ for such pairs.)

Let $\omega=\left(j_{2}^{1}, \ldots, j_{2}^{r}\right) \in \Omega_{j_{2}}^{s+1}$ for some $j_{2} \in J_{2}^{1} \cup \cdots \cup J_{2}^{s}$ and let $p \in$ $\mathscr{P}_{j_{2}}$. If $p=Q_{\gamma}$ for some $\gamma \in \Gamma_{1} \cup \cdots \cup \Gamma_{s}$, then obtain a set of data as in (6.5.1) by letting $v_{0}=1, v_{1}=s+1(l=1)$, and $\left(j_{2}^{1_{1}}, \ldots, j_{2}^{r_{1}}\right)=\omega$; extract the data of (6.5.2) from the above data and alter $H_{1, s+1}^{\prime}$ as outlined in (6.5) (more precisely, complete that part of the alteration which has not been done previously). If $p=Q_{\omega_{2}^{\prime} p_{2}^{\prime}}$ (it is convenient to let $\omega_{1}^{\prime}=\omega, p_{1}^{\prime}=p, v_{1}^{\prime}=s+1$, and $j_{2,1}=j_{2}$ ), then integers $1 \leqslant v_{0}<v_{1} \lesseqgtr \cdots \leqslant v_{t}<I(\mathbb{Q})$, chains $\left(j_{2}^{1}, \ldots, j_{2}^{r_{t}}\right)$ for $l=1, \ldots, t$, and an element $\underset{\gamma}{\gamma}=\left(j_{2}^{\prime}, \stackrel{\neq}{u_{k}}\right) \in \Gamma_{v_{0}}$ are uniquely determined as follows. Let $v_{2}^{\prime}$ be such that $\omega_{2}^{\prime} \in \Omega_{j_{2,2}}^{v_{2}^{\prime}}$ for some $j_{2,2} \in J_{2}^{1}$ $\cup \cdots \cup J_{2}^{v_{2}^{\prime}-1}$ (necessarily, $\left.v_{2}^{\prime} \lesseqgtr v_{1}^{\prime}\right)$. If $p_{2}^{\prime}=Q_{\omega_{3}^{\prime} p_{3}^{\prime}}$, then let $v_{3}^{\prime}$ be such that

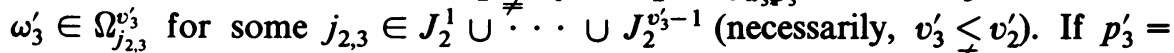
$Q_{\omega_{4}^{\prime} p_{4}^{\prime}}$, then let $v_{4}^{\prime}$ be such that $\omega_{4}^{\prime} \in \Omega_{j_{2,4}}^{v_{4}^{\prime}}$ for some $j_{2,4} \in J_{2}^{1} \cup^{\neq} \cdots \cup J_{2}^{v_{4}^{\prime}-1}$ (necessarily, $v_{4}^{\prime} \lesseqgtr v_{3}^{\prime}$ ). Continue in this manner until $p_{t}^{\prime}=Q_{\omega_{+1}^{\prime} p_{t+1}^{\prime}}$ and $p_{t+1}^{\prime}=$

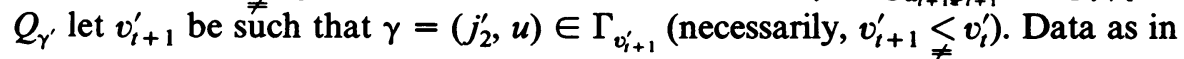
(6.5.1) is obtained by letting $v_{0}=v_{t+1}^{\prime}, v_{1}=v_{t}^{\prime}, v_{2}=v_{t-1}^{\prime}, \ldots, v_{t}=v_{1}^{\prime}$ and letting $\left(j_{2}^{1}, \ldots, j_{2}^{r_{t}}\right)=\omega_{t-l+1}^{\prime}$ for $l=1, \ldots, t$. Extract the data of (6.5.2) from the above data and alter $H_{1, s+1}^{\prime}$ as outlined in (6.5) (more precisely, complete that part of the alteration which has not been done previously).

Let $H_{1, s+1}$ be the mapping obtained by making the above alteration for all triples $\omega \in \Omega_{j_{2}}^{s+1}, j_{2} \in J_{2}^{1} \cup \cdots \cup J_{2}^{s}$, and $p \in \mathscr{P}_{j_{2}}$. Certainly, $H_{1, s+1}$ satisfies $(6.1 .2)^{2}$ and $(6.1 .5)^{2}$ for $j_{2} \in J_{2}^{1} \cup \cdots \cup J_{2}^{s^{2+1}}$ and there is no difficulty in doing the above altering of $H_{1, s+1}^{\prime}$ so that $H_{1, s+1}$ satisfies (6.1.4) for $j_{2}, j_{2}^{\prime} \in J_{2}^{1} \cup \cdots \cup J_{2}^{s}$. That $(6.1 .4)^{2}$ holds for pairs $j_{2}, j_{2}^{\prime} \in J_{2}^{1} \cup \cdots \cup$ $J_{2}^{s+1}$ can be checked by checking the following cases.

Case 1.1. $j_{2} \in J_{2}^{s+1}$ and $j^{\prime} \in J_{2}^{1}$ with $j_{2} \cap j_{2}^{\prime} \neq \varnothing$.

Case 1.2. $j_{2} \in J_{2}^{s+1}$ and $j_{2}^{\prime} \in J_{2}^{2}$ with $j_{2} \cap j_{2}^{\prime} \neq \varnothing$.

Case 1.s. $j_{2} \in J_{2}^{s+1}$ and $j_{2}^{\prime} \in J_{2}^{s}$ with $j_{2} \cap j_{2}^{\prime} \neq \varnothing$.

Case 2. $j_{2}, j_{2}^{\prime} \in J_{2}^{s+1}$ with $j_{2} \cap j_{2}^{\prime} \neq \varnothing$.

Checking Case 1.e for $1 \leqslant e \leqslant s$. Let $y \in \Re_{e}$ with $j_{2}^{\prime} \in J_{2}^{y}$ and let $x \in$ $\Re_{s+1}$ with $j_{2} \in J_{2}^{x}$. Let $x=\left(u_{1}, \ldots, u_{k}\right)$ and $y=\left(u_{1}^{\prime}, \ldots, u_{k^{\prime}}^{\prime}\right)$; it follows from $(6.3 .1)_{s+1}$ that $x \geqslant y$ and, therefore, that $\left\{u_{1}, \ldots, u_{k}\right\} \subseteq\left\{u_{1}^{\prime}, \ldots, u_{k^{\prime}}^{\prime}\right\}$.

$H_{1, s+1}^{-1}\left(\operatorname{int}\left(j_{2}\right)\right) \subseteq N_{\delta}\left(H_{1, s+1}^{-1}\left(\operatorname{int}\left(j_{2}^{\prime}\right)\right)\right)$. It suffices to show that if $H_{1, s+1}^{-1}\left(\operatorname{int}\left(j_{2}\right)\right) \cap H_{1}^{-1}\left(\operatorname{int}\left(j_{1}^{u}\right)\right) \neq \varnothing$, then this intersection is contained in $N_{\delta / 2}\left(H_{1, s+1}^{-1}\left(\operatorname{int}\left(j_{2}^{\prime}\right)\right)\right)$. If $u \in\left\{u_{1}^{\prime}, \ldots, u_{k^{\prime}}^{\prime}\right\}$, then the latter containment holds since $H_{1}^{-1}\left(\operatorname{int}\left(j_{1}^{u}\right)\right) \subseteq N_{\delta / 2}\left(H_{1, s+1}^{-1}\left(\operatorname{int}\left(j_{2}^{\prime}\right)\right)\right)$. For $u \notin\left\{u_{1}^{\prime}, \ldots, u_{k^{\prime}}^{\prime}\right\}$, our approach is to show that if an alteration of $H_{1, s}$ resulted in $H_{1, s}^{-1}\left(\operatorname{int}\left(j_{2}\right)\right)$ being "pulled into" $H_{1}^{-1}\left(\operatorname{int}\left(j_{1}^{u}\right)\right)$, then that part of $H_{1, s+1}^{-1}\left(\operatorname{int}\left(j_{2}\right)\right) \cap H_{1}^{-1}\left(\operatorname{int}\left(j_{1}^{u}\right)\right)$ 
resulting from the alteration is contained in $N_{\delta / 2}\left(H_{1, s+1}^{-1}\left(\operatorname{int}\left(j_{2}^{\prime}\right)\right)\right)$.

The set $H_{1, s+1}^{-1}\left(\operatorname{int}\left(j_{2}\right)\right) \cap H_{1}^{-1}\left(\operatorname{int}\left(j_{1}^{u}\right)\right)$ is determined by alterations made with respect to sets of data consisting of integers $1 \leqslant v_{0}<v_{1}<\cdots<v_{t}=s+1, \gamma=\left(j_{2}^{\prime \prime}, u^{\prime \prime}\right) \in \Gamma_{v_{0}}$, and chains $\left(j_{2}^{1}, \ldots, j_{2}^{r_{t}}\right)$ for $l=1, \ldots, t$ satisfying (6.5.1) such that $j_{2}$ appears in $\left(j_{2}^{1_{1}}, \ldots, j_{2}^{r_{t}}\right)$ and one or both of the following hold: (i) various of the $j_{2}^{i}$ 's are subsets of $j_{1}^{u}$; (ii) $u^{\prime \prime}=u$.

Let $q$ be such that $1 \leqslant v_{0} \lesseqgtr v_{1} \lesseqgtr \cdots<v_{q} \leqslant e \lesseqgtr v_{q+1} \lesseqgtr \cdots<v_{\text {, }}$. Exploiting the fact that $u \notin\left\{u_{1}^{\prime}, \ldots, u_{k^{\prime}}^{\neq}\right\}$we will now show that $j_{2}^{\prime} \cap j_{2}^{1} \not \neq \varnothing$ and that if $j_{2}^{i_{l}} \subseteq j_{1}^{u}$, then $1 \leqslant l \leqslant q-1$. Condition (6.3.3) implies that $j_{2}^{\prime} \cap j_{2}^{r_{t-1}} \neq \varnothing$ (the reason being that both $j_{2}^{\prime}$ and $j_{2}^{r_{t-1}}$ meet $\mathbf{s t}^{\mathcal{E}_{\mathrm{v}_{t}-1}}\left(j_{2}, J_{2}^{v_{t}}\right)^{*}$ and $\left.J_{2}^{v_{t}} \subseteq \mathcal{G}_{c_{2}^{v_{1}}}\right) ; j_{2}^{\prime} \cap j_{2}^{r_{t-1}} \neq \varnothing$ and condition (6.3.1) $)_{v_{t-1}}$ imply that if $z \in \Re_{v_{t-1}}$ with $j_{2}^{r_{t-1}} \in J_{2}^{z}$, then $z \geqslant y$; if $z^{\prime} \in \Re_{v_{t-1}}$ and $z^{\prime} \neq z$, then (6.3.1) $)_{v_{t-1}}$ implies that $\left(J_{2}^{z^{\prime}}\right)^{*} \cap\left(J_{2}^{z}\right)^{*}=\varnothing$ and, therefore,

$$
\left\{j_{2}^{1_{v_{t}-1}}, \ldots, j_{2}^{r_{v_{t}-1}}\right\}^{*} \subseteq\left(J_{2}^{z}\right)^{*} \subseteq j_{1}^{u_{1}^{\prime}} \cup \cdots \cup j_{1}^{u_{k^{\prime}}} .
$$

We can use the argument in the preceding sentence to proceed inductively for $i=t-1, t-2, \ldots, q+1$ as follows. Since $j_{2}^{\prime} \cap j_{2}^{r_{i}} \neq \varnothing$, condition (6.3.3) implies that $j_{2}^{\prime} \cap j_{2}^{r_{i-1}} \neq \varnothing$; condition (6.3.1) $)_{v_{i-1}}$ and $j_{2}^{\prime} \cap j_{2}^{r_{i-1}} \neq \varnothing$ imply that if $z \in \Re_{v_{i-1}}$ with $j_{2}^{r_{i-1}} \in J_{2}^{z}$, then $z \geqslant y$; if $z^{\prime} \in \mathscr{N}_{v_{i-1}}$ and $z^{\prime} \neq z$, then $(6.3 .1)_{v_{i-1}}$ implies that $\left(J_{2}^{z^{\prime}}\right)^{*} \cap\left(J_{2}^{z}\right)^{*}=\varnothing$ and, therefore,

$$
\left\{j_{2}^{1_{v_{i}-1}}, \ldots, j_{2}^{r_{v_{i-1}}}\right\}^{*} \subseteq\left(J_{2}^{z}\right)^{*} \subseteq j_{1}^{u_{1}^{\prime}} \cup \cdots \cup j_{1}^{u_{k^{\prime}}} .
$$

If $v_{q}=e$ and $j_{2}^{\prime} \in\left\{j_{2}^{1}, \ldots, j_{2}^{r_{q}}\right\}$, then that part of $H_{1, s}^{-1}\left(\operatorname{int}\left(j_{2}\right)\right)$ being "pulled into" $H_{1}^{-1}\left(\operatorname{int}\left(j_{1}^{u}\right)\right)$ by the alteration based on the data $\gamma=\left(j_{2}^{\prime \prime}, u^{\prime \prime}\right) \in$ $\Gamma_{v_{0}}$ and $\left\{\left(j^{1}, \ldots, j^{r_{l}}\right)\right\}_{l=1, \ldots, t}$ will be "pulled inside" of $H_{1, s}^{-1}\left(\operatorname{int}\left(j_{2}^{\prime}\right)\right) \cap$ $H_{1}^{-1}\left(\operatorname{int}\left(j_{1}^{u}\right)\right)$ and, therefore, will be contained in $N_{\delta / 2}\left(H_{1, s+1}^{-1}\left(\operatorname{int}\left(j_{2}^{\prime}\right)\right)\right)$.

If $v_{q}=e, j_{2}^{\prime} \notin\left\{j_{2}^{1}, \ldots, j_{2}^{r_{q}}\right\}$, and $r_{q}<\mathcal{E}_{v_{q}}$, then that part of $H_{1, s}^{-1}\left(\operatorname{int}\left(j_{2}\right)\right)$ being "pulled into" $H_{1}^{-1}\left(\operatorname{int}\left(j_{1}^{u}\right)\right)$ based on the data $\gamma=\left(j_{2}^{\prime \prime}, u^{\prime \prime}\right) \in \Gamma_{v_{0}}$ and $\left\{\left(j_{2}^{1}, \ldots, j_{2}^{{ }^{\prime}}\right)\right\}_{l=1, \ldots, t}$ will be "pulled alongside" that part of $H_{1,5}^{-1}\left(\operatorname{int}\left(j_{2}^{\prime}\right)\right)$ which was "pulled into" $H_{1}^{-1}\left(\operatorname{int}\left(j_{1}^{u}\right)\right)$ by the previous alteration (done when altering $H_{1, v_{q}-1}$ to get $\left.H_{1, v_{q}}\right)$ based on the data $\gamma=\left(j_{2}^{\prime \prime}, u^{\prime \prime}\right) \in \Gamma_{v_{0}}$ and $\left\{\left(j_{2}^{1}, \ldots, j_{2}^{r_{l}}\right) \mid l=1, \ldots, q-1\right\} \cup\left\{\left(j_{2}^{1}{ }^{1}, \ldots, j_{2}^{r_{q}}, j_{2}^{\prime}\right)\right\}$. In particular, we will have the part of $H_{1, s+1}^{-1}\left(\operatorname{int}\left(j_{2}\right)\right) \cap H_{1}^{-1}\left(\operatorname{int}\left(j_{1}^{u}\right)\right)$ resulting from the above alteration contained in $N_{\delta / 2}\left(H_{1, s+1}^{-1}\left(\operatorname{int}\left(j_{2}^{\prime}\right)\right)\right)$.

If $v_{q}=E, j_{2}^{\prime} \notin\left\{j_{2}^{1}, \ldots, j_{2}^{r_{q}}\right\}$, and $r_{q}=\mathcal{E}_{v_{q}}$, then the alteration based on the data $\gamma=\left(j_{2}^{\prime \prime}, u^{\prime \prime}\right) \in \Gamma_{v_{0}}$ and $\left\{\left(j_{2}^{1}, \ldots, j_{2}^{{ }^{\prime}}\right)\right\}_{l=1, \ldots, t}$ will not result in $H_{1, s}^{-1}\left(\operatorname{int}\left(j_{2}\right)\right)$ being "pulled into" $H_{1}^{-1}\left(\operatorname{int}\left(j_{1}^{u}\right)\right)$. (To see this, recall that none of the $j_{2}^{i}$,s for $l=q, q+1, \ldots, t$ are contained in $j_{1}^{u}$ and, using the definition of $\varepsilon_{v_{q}}$, show that the part of $H_{1, s+1}^{-1}\left(\operatorname{int}\left(j_{2}^{r_{q}}\right)\right)$ into which $H_{1, s}^{-1}\left(\operatorname{int}\left(j_{2}\right)\right)$ is being pulled does not meet $H_{1}^{-1}\left(\operatorname{int}\left(j_{1}^{u}\right)\right)$.) 
If $v_{q} \neq e$, then the part of $H_{1, s}^{-1}\left(\operatorname{int}\left(j_{2}\right)\right)$ being "pulled into" $H_{1}^{-1}\left(\operatorname{int}\left(j_{1}^{u}\right)\right)$ based on the data $\gamma=\left(j_{2}^{\prime \prime}, u^{\prime \prime}\right) \in \Gamma_{v_{0}}$ and $\left\{\left(j_{2}^{1}, \ldots, j_{2}^{\prime \prime}\right)\right\}_{l=1, \ldots, t}$, will be "pulled alongside" that part of $H_{1, s}^{-1}\left(\operatorname{int}\left(j_{2}^{\prime}\right)\right)$ which was "pulled into" $H_{1}^{-1}\left(\operatorname{int}\left(j_{1}^{u}\right)\right)$ by the previous alteration (done when altering $H_{1, v_{q}-1}$ to get $\left.H_{1, v_{q}}\right)$ based on the data $\gamma=\left(j_{2}^{\prime \prime}, u^{\prime \prime}\right) \in \Gamma_{v_{0}}$ and $\left\{\left(j_{2}^{1}, \ldots, j_{2}^{r_{1}}\right) \mid l=1, \ldots, q\right\}$ $\cup\left\{\left(j_{2}^{\prime}\right)\right\}$.

The arguments given in the preceeding paragraphs combine to show that $H_{1, s+1}^{-1}\left(\operatorname{int}\left(j_{2}\right)\right) \subseteq N_{\delta}\left(H_{1, s+1}^{-1}\left(\operatorname{int}\left(j_{2}^{\prime}\right)\right)\right)$.

$H_{1, s+1}^{-1}\left(\operatorname{int}\left(j_{2}^{\prime}\right)\right) \subseteq N_{\delta}\left(H_{1, s+1}^{-1}\left(\operatorname{int}\left(j_{2}\right)\right)\right)$. If $u \in\left\{u_{1}, \ldots, u_{k}\right\}$, then

$$
H_{1, s+1}^{-1}\left(\operatorname{int}\left(j_{2}^{\prime}\right)\right) \cap H_{1}^{-1}\left(\operatorname{int}\left(j_{1}^{u}\right)\right) \subseteq N_{\delta / 2}\left(H_{1, s+1}^{-1}\left(\operatorname{int}\left(j_{2}^{\prime}\right)\right)\right)
$$

since

$$
H_{1}^{-1}\left(\operatorname{int}\left(j_{1}^{u}\right)\right) \subseteq N_{\delta / 2}\left(H_{1, s+1}^{-1}\left(\operatorname{int}\left(j_{2}\right)\right)\right) .
$$

If $u \notin\left\{u_{1}, \ldots, u_{k}\right\}$, then $H_{1, s+1}^{-1}\left(\operatorname{int}\left(j_{2}^{\prime}\right)\right) \cap H_{1}^{-1}\left(\operatorname{int}\left(j_{1}^{u}\right)\right)$ is determined by alterations made with respect to sets of data consisting of integers $1 \leqslant v_{0}<v_{1} \lesseqgtr \cdots<v_{t}=e, \gamma=\left(j_{2}^{\prime \prime}, u^{\prime \prime}\right)<\Gamma_{v_{0}}$, and chains $\left(j_{2}^{1}, \ldots, j_{2}^{\prime \prime}\right)$ for $l=1, \ldots, t$ satisfying (6.5.1) such that $j_{2}^{\prime}$ appears in $\left(j_{2}^{1}, \ldots, j_{2}^{r^{\prime}}\right)$, say $j_{2}^{\prime}=j_{2}^{q_{1}}$, and one or both of the following hold: (i) various of the $j_{2}^{i}$ 's are subsets of $j_{1}^{u}$; (ii) $u^{\prime \prime}=u$. That part of $H_{1, s+1}^{-1}\left(\operatorname{int}\left(j_{2}^{\prime}\right)\right) \cap H_{1}^{-1}\left(\operatorname{int}\left(j_{1}^{u}\right)\right)$ resulting from an alteration based on such a set of data will be contained in $N_{\delta / 2}\left(H_{1, s+1}^{-1}\left(\operatorname{int}\left(j_{2}\right)\right)\right)$ since $H_{1, s}^{-1}\left(\operatorname{int}\left(j_{2}\right)\right)$ will be "pulled inside" by the alteration based on the data consisting of integers $1 \leqslant v_{0} \lesseqgtr v_{1} \lesseqgtr \cdots v_{t}=e \lesseqgtr s+1, \gamma=\left(j_{2}^{\prime \prime}, u^{\prime \prime}\right) \in \Gamma_{v_{0}}$, and chains $\left\{\left(j_{2}^{1}, \ldots, j_{2}^{1^{\prime}}\right) \mid l=1, \ldots, t-1\right\} \cup\left\{\left(j_{2}^{1}, \ldots, j_{2}^{q_{1}}\right)\right\} \cup\left\{\left(j_{2}\right)\right\}$. Consideration of Case 1.e is now completed.

Checking Case 2. $j_{2}, j_{2}^{\prime} \in J_{2}^{s+1}$ with $j_{2} \cap j_{2}^{\prime} \neq \varnothing$.

Since the roles of $j_{2}$ and $j_{2}^{\prime}$ are interchangeable, it suffices to show that $H_{1, s+1}^{-1}\left(\operatorname{int}\left(j_{2}\right)\right) \subseteq N_{\delta}\left(H_{1, s+1}^{-1}\left(\operatorname{int}\left(j_{2}^{\prime}\right)\right)\right)$. First, observe that (6.3.1 $)_{s+1}$ implies that there is a unique $y=\left(u_{1}, \ldots, u_{k}\right) \in \Re_{s+1}$ with $j_{2}, j_{2}^{\prime} \in J_{2}^{y}$. For $i=$ $1, \ldots, k$, we have that

$$
H_{1, s+1}^{-1}\left(\operatorname{int}\left(j_{2}\right)\right) \cap H_{1}^{-1}\left(\operatorname{int}\left(j_{1}^{u_{i}}\right)\right) \subseteq H_{1}^{-1}\left(\operatorname{int}\left(j_{1}^{\mu_{i}}\right)\right) \subseteq N_{\delta / 2}\left(H_{1, s+1}^{-1}\left(\operatorname{int}\left(j_{2}^{\prime}\right)\right)\right) .
$$

For $u \notin\left\{u_{1}, \ldots, u_{k}\right\}$ with $H_{1, s+1}^{-1}\left(\operatorname{int}\left(j_{2}\right)\right) \cap H_{1}^{-1}\left(\operatorname{int}\left(j_{1}^{u}\right)\right) \neq \varnothing$, we must show that the intersection is contained in $N_{\delta / 2}\left(H_{1, s+1}^{-1}\left(\operatorname{int}\left(j_{2}^{\prime}\right)\right)\right.$ ). This intersection is determined by alterations made with respect to sets of data consisting of integers $1<v_{0} \lesseqgtr v_{1} \lesseqgtr \cdots \lesseqgtr v_{t}=s+1, \gamma=\left(j_{2}^{\prime \prime}, u^{\prime \prime}\right) \in \Gamma_{v_{0}}$, and chains $\left(j_{2}^{1}, \ldots, j_{2}^{t^{\prime}}\right)$ for $l \stackrel{\neq}{=}, \ldots, t$ satisfying (6.5.1) such that $j_{2}=j_{2}^{t^{\prime}}$ and one or both of the following hold: (i) various of the $j_{2}^{i}$,s are subsets of $j_{1}^{\mu}$; (ii) $u^{\prime \prime}=u$. (Recall that $r_{t}<\mathcal{E}_{s}$.) If $r_{t}=\mathcal{E}_{s}$, then the alteration based on the above data will not result in $H_{1, s+1}^{-1}\left(\operatorname{int}\left(j_{2}\right)\right)$ meeting $H_{1}^{-1}\left(\operatorname{int}\left(j_{1}^{u}\right)\right)$; the reader 
can check this statement by using the definition of $\mathcal{E}_{s}$ and an argument similar to that used in considering Case 2 for $s=2$. If $r<\mathcal{E}_{s}$, then either $j_{2}^{\prime}=j_{2}^{i}$ for some $1 \leqslant i_{t} \leqslant r_{t}-1$ or, if such is not the case, then $H_{1, s}^{-1}\left(\operatorname{int}\left(j_{2}^{\prime}\right)\right)$ will be altered with respect to the data consisting of the integers $1 \leqslant v_{0} \lesseqgtr v_{1} \lesseqgtr \cdots v_{t}=s+1, \gamma=\left(j_{2}^{\prime \prime}, u^{\prime \prime}\right) \in \Gamma_{v_{0}}$, and chains $\left\{\left(j_{2}^{1}, \ldots, j_{2}^{r_{t}}\right) \mid l=1, \ldots, t-1\right\} \cup\left\{\left(j_{2}^{1}, \ldots, j_{2}^{r_{t}}=j_{2}, j_{2}^{\prime}\right)\right\}$. We leave to the reader to check that in either case the desired containment holds.

(6.7) Letting $H_{2}=H_{1, I(\mathscr{Q})}$, the only condition which remains to be verified is (6.1.3) $)^{2}$. The reader should first study the effect of the alterations described in (6.5) in order to verify that if $j_{2} \in J_{2}$ and $H_{2}^{-1}\left(\operatorname{int}\left(j_{2}\right)\right) \cap H_{1}^{-1}\left(\operatorname{int}\left(j_{1}\right)\right) \neq \varnothing$ for some $j_{1} \in J_{1}$, then $j_{2} \in \mathrm{st}^{\mathcal{G}_{1(())-1}}\left(j_{1}, J_{2}\right)$. Conditions $(6.3 .2)_{s}, s=$ $2, \ldots, I(\mathscr{Q})$, imply that $\operatorname{st}^{\mathcal{E}_{I(\Theta)}}\left(j_{1}, J_{2}\right)^{*} \subseteq \operatorname{int}\left(\operatorname{st}\left(j_{1}, \mathcal{G}_{a_{2}}\right)^{*}\right)$; the latter containment and condition (6.2.1) imply (6.1.3) ${ }^{2}$ as follows. Let $g \in \mathcal{G}_{a_{2}}$ be such that $j_{2} \subseteq g$; hence, $\operatorname{st}\left(j_{2}, J_{2}\right)^{*} \subseteq \operatorname{st}^{2}\left(g, \mathcal{G}_{a_{2}}\right)^{*}$. If $j_{1} \in J_{1}$ is such that $H_{2}^{-1}\left(\operatorname{int}\left(j_{2}^{\prime}\right)\right) \cap$ $H_{1}^{-1}\left(\operatorname{int}\left(j_{1}\right)\right) \neq \varnothing$ for some $j_{2}^{\prime} \in \operatorname{st}\left(j_{2}, J_{2}\right)$, then $j_{1}$ meets $\operatorname{st}^{2}\left(g, \mathcal{G}_{a_{2}}\right)^{*}$ since $j_{2}^{\prime} \in \operatorname{st}^{\mathcal{E}_{l(\alpha)}}\left(j_{1}, J_{2}\right)^{*} \subseteq \operatorname{int}\left(\operatorname{st}\left(j_{1}, \mathcal{G}_{a_{2}}\right)^{*}\right)$. Condition $(6.1 .3)^{2}$ now follows from (6.2.1). This completes the construction of the $n=2$ stage.

(6.8) The construction of the $n+1$ st stage from the $n$th stage is done exactly as the construction of the 2 nd stage from the 1st stage was done provided the following change of parameters is made in (6.2). Let $\delta=1 / 2^{n+1}$ and let $a_{n+1}$ be such that $\mathcal{G}_{a_{n+1}}$ refines each of the $\mathcal{G}_{i}$ 's used during the construction of the 1st through $n$th stages. In place of $\xi_{2} / 2^{2}$ use $\xi_{n+1} / 2^{n+1}$, in place of $J_{1}$ use $J_{n}$, and in place of $H_{1}$ use $H_{n}$. This completes the proof of Proposition (6.1).

Proof of THE MaIn Theorem.

(6.9) Recall that in $\$ 1$ we reduced the Main Theorem to the case with $f$ monotone. Using Proposition (6.1) construct triples $R_{n}: J_{n} \rightarrow P_{n}$ by letting $P_{n}=\left\{\operatorname{cl}\left(H_{n}^{-1}\left(\operatorname{int}\left(j_{n}\right)\right)\right) \mid j_{n} \in J_{n}\right\}$ and letting $R_{n}\left(j_{n}\right)=\operatorname{cl}\left(H_{n}^{-1}\left(\operatorname{int}\left(j_{n}\right)\right)\right)$. It is easily verified that the sequence of triples satisfies the hypothesis of Proposition (5.2). Let $g$ be the open mapping obtained from Proposition (5.1) and, as in (4.3), it follows that $g=\lim _{n \rightarrow \infty} H_{n}$. (We leave to the reader to extract the isotopy $\left\{h_{t}\right\}_{t \in[0, \infty)}$ from the proof of Proposition (6.1).) Finally, to be sure that each $g^{-1}(y)$ is nondegenerate modify the proof of Proposition (6.1) exactly as the proof of Proposition (4.2) was modified in (4.3).

\section{REFERENCES}

[A-1]. R. D. Anderson, Open mappings of compact continua, Proc. Nat. Acad. Sci. U.S.A. 42 (1956), 347-349. MR 17, 1230.

[A-2]. , Monotone interior dimension raising mappings, Duke Math. J. 19 (1952), 359-366. MR 14, 71.

[A-3]. , Continuous collections of continuous curves, Duke Math. J. 21 (1954), 363-367. MR 15, 977 . 
$[A-4]$. 211-222. On monotone interior mappings in the plane, Trans. Amer. Math. Soc. 73 (1952),

[Bi]. R. H. Bing, Complementary domains of contimuous curves, Fund. Math. 36 (1949), 303-318. MR 12, 348.

[Ke]. L. V. Keldys, Transformation of a monotone irreducible mapping into a monotone open one and monotone mappings of a cube which raise dimension, Dokl. Akad. Nauk SSSR 114 (1957), 472-475. (Russian) MR 19, 972.

[K-L]. K. Kuratowski and C. Lacher, $A$ theorem on the space of monotone mappings, Bull. Acad. Polon. Sci. Sér. Sci. Math. Astronom. Phys. 17 (1969), 797-800. MR 43 \# 1143.

[McA]. L. F. McAuley, Open mappings and open problems, Topology Conf., Arizona State Univ., Tempe, Ariz., 1968, pp. 184-202. MR 39 \#2134.

[So]. A. B. Sosinskii, Monotonically-open mappings of a sphere,, Amer. Math. Soc. Transl. (2) 78 (1968), 67-101.

[Wa-1]. J. J. Walsh, Monotone and open mappings on manifolds. I, Trans. Amer. Math. Soc. 209 (1975), 419-432.

[Wa-2]. __ Light open and open mappings on manifolds. II, Trans. Amer. Math. Soc. 217 (1976), 271-284.

[Wa-3]. Monotone and open mappings onto ANR's, Proc. Amer. Math. Soc. 60 (1976), 286-288.

[Wi-1]. D. C. Wilson, Open mappings on manifolds and a counterexample to the Whyburn conjecture, Duke Math. J. 40 (1973), 705-716. MR 47 \#9522.

[Wi-2]. Open mappings of the universal curve onto continuous curves, Trans. Amer. Math. Soc. 168 (1972), 487-515. MR 45 \# 7682.

[Why-1]. G. T. Whyburn, Analytic topology, Amer. Math. Soc. Colloq. Publ., vol. 28, Amer. Math. Soc., Providence, R. I., 1942.

[Why-2]. Topological analysis, Princeton Univ. Press, Princeton, N. J., 1958.

Department of Mathematics, University of Oxtahoma, Norman, OkIahoma 73069

Current address: Department of Mathematics, University of Tennessee, Knoxville, Tennessee 37916 